\title{
Efecto de los estadios sucesionales del bosque tropical seco sobre el microhábitat usado por Agalychnis dacnicolor (Anura: Phyllomedusidae) y Smilisca fodiens (Anura: Hylidae)
}

\author{
Yunuen Soto-Sandoval ${ }^{1}$, Ireri Suazo-Ortuño ${ }^{1 *}$, Nicolás Urbina-Cardona ${ }^{2}$, \\ Jorge Marroquín-Páramo ${ }^{1} \&$ Javier Alvarado-Díaz ${ }^{1}$ \\ 1. Instituto de Investigaciones sobre los Recursos Naturales, Universidad Michoacana de San Nicolás de Hidalgo. Av. \\ San Juanito Itzícuaro s/n, Col. Nueva Esperanza, Morelia, Michoacán, CP 58330, México; \\ yun_biol_11@hotmail.com, ireri.suazo@gmail.com, jorgemapa24@gmail.com, jvr.alvarado@gmail.com \\ 2. Department of Ecology and Territory, School of Rural and Environmental Studies, Pontificia Universidad Javeriana, \\ Bogota- Colombia; urbina-j@javeriana.edu.co \\ * Correspondence
}

Recibido 31-V-2016. Corregido 10-I-2017. Aceptado 26-I-2017.

\begin{abstract}
Effect of tropical dry forest succesional stages on microhabitat use by Agalychnis dacnicolor (Anura: Phyllomedusidae) and Smilisca fodiens (Anura: Hylidae). Habitat disturbance can modify microhabitats and therefore have a significant effect on the abundance and distribution of anurans species, particularly those associated to specific microhabitats. In this study we evaluated changes in the use of microhabitat by two syntopic species of tree frogs, Agalychnis dacnicolor and Smilisca fodiens along a successional gradient of Tropical Dry Forest in the region of the Biosphere Reserve of Chamela-Cuixmala at the coast of Jalisco, Mexico. We hypothesized that because these two species are habitat generalists, microhabitat use would change in response to secondary forest succession and to the climatic season of the year. Although both species were registered in both secondary and mature forests, A. dacnicolor was associated to mature forest, whereas $S$. fodiens was more associated to disturbed sites. Neither species showed differences in biomass among successional stages (ES) or between climatic seasons. Unlike $S$. fodiens, A. dacnicolor presented larger sizes during the rainy season. Body temperature of both species varied between ES and climatic season. S. fodiens presented a more constant temperature among individuals in pastures, while $A$. dacnicolor in mature forests. Environmental and structural habitat variables that influenced the presence of both species in ES were substrate temperature, height of tree or shrub where the frog was perching and percentage cover of litter, grasses, shrubs, trees and bare soil. The variables that best explained biomass, snout-vent length (LHC) and body temperature of $S$. fodiens were cover of dry branches and substrate temperature, whereas biomass of $A$. dacnicolor was better explained by cover of herbs, LHC, height of the tree or shrub where the frog was perching, body temperature and the substrate temperature. Both species used differentially environmental and structural variables of microhabitats along the gradient of initial to late ES. The variables that separated the species were the height of the shelter or perch and the percentage of trees in different ES. Whereas A. dacnicolor was more associated to the lowest strata of vegetation, $S$. fodiens showed plasticity in microhabitat use, especially in height of shelter or perch. Our results suggested that even when both species are considered habitat generalists, in anthropic landscapes, A. dacnicolor is specialist in microhabitat use, whereas $S$. fodiens is a species with greater ecological plasticity in microhabitat use. These differences in microhabitat use in response to habitat disturbance may help explain the success of these species in these new landscapes, as well as the importance of secondary forests in different stages of succession to maintain adequate microhabitats for the permanence of species in anthropogenic landscapes. Rev. Biol. Trop. 65 (2): 777-798. Epub 2017 June 01.
\end{abstract}

Key words: Hylidae, chronosequence, habitat features, secondary forest. 
A nivel global las poblaciones de anfibios están experimentando un declive masivo, algunas de las causas son la introducción de especies exóticas, enfermedades emergentes, contaminantes químicos, cambio climático global y la transformación del hábitat ya sea por degradación, pérdida o fragmentación (Blaustein et al., 2011). Los anfibios son un grupo susceptible a la degradación del ambiente por sus características morfológicas y fisiológicas, como el tamaño corporal relativamente pequeño, que influye en la relación superficie-volumen y por consiguiente en la susceptibilidad a la desecación y a la dependencia de la temperatura del medio para mantener su cuerpo en rangos de temperatura adecuados para su desempeño; y piel delgada y semipermeable que los hace más vulnerables a efectos negativos de diversos agentes químicos y cambios en el microclima (Vitt \& Caldwell, 2014). Estas características hacen que los anfibios sean sensibles a cambios en las características del hábitat. Sin embargo, la mayoría de los hábitats en los que los anfibios se presentan están siendo transformados por actividades humanas a un ritmo acelerado. Tal es el caso de los bosques tropicales secos (BTS), reconocidos como uno de los hábitats más amenazados del mundo (Janzen, 1988; Dirzo, Young, Mooney \& Ceballos, 2011). México cuenta con el $38 \%$ de la extensión total de BTS en el Continente Americano, lo cual representa una extensión de $181461 \mathrm{~km}^{2}$. Sin embargo, solo el $27 \%$ de su cobertura original permanece intacta (Trejo \& Dirzo, 2000; Portillo-Quintero \& Sánchez-Azofeifa, 2010) y a pesar de su importancia biológica, se estima que al menos el $48 \%$ de la extensión de estos bosques se ha perdido debido al cambio de uso del suelo (Dirzo et al., 2011).

De acuerdo con varios reportes, la perturbación de los bosques altera la composición y estructura de las comunidades herpetofaunísticas debido a la modificación o desaparición de las condiciones ambientales y estructurales de los microhábitats presentes en bosques no perturbados (Urbina-Cardona \& Londoño, 2003; Urbina-Cardona, 2008). La manera como las especies de anfibios responden a los disturbios del hábitat depende en gran medida de su habilidad de adaptación y de sus características poblacionales (Hunter, 1996). Los anfibios dependen de microhábitats que les provean las condiciones adecuadas para refugio, alimentación, reproducción, estivación y escape de depredadores, entre otros aspectos (UrbinaCardona \& Reynoso, 2011; Urbina-Cardona, Reynoso \& Dirzo, 2012). Sin embargo, la mayoría de las especies de anfibios han mostrado ser especialmente sensibles a cambios drásticos en sus microhábitats (Duellman \& Trueb, 1994). Los rangos estrechos de tolerancia ecofisiológica de los anfibios, hace que estos sean abundantes en áreas con condiciones de microhábitat óptimas, pero cuando estas condiciones se ven modificadas aumenta su vulnerabilidad dependiendo de la sensibilidad de cada especie (Pizano \& García, 2014; Urbina-Cardona et al., 2014). Diversos estudios han propuesto que el microhábitat de las especies se modifica tras el paso de alguna perturbación (Isaacs-Cubides \& Urbina-Cardona, 2011; Cortés-Gómez, CastroHerrera \& Urbina-Cardona, 2013).

Un resultado de la perturbación de los BTS, es el establecimiento de bosques secundarios. Estos presentan una composición y estructura vegetal diferente a la del bosque primario, así como cambios estructurales importantes a lo largo del proceso de sucesión (Morales \& Sarmiento, 2002). Por ejemplo, los bosques secundarios presentan diferencias significativas entre los estadios sucesionales en cuanto a la densidad de árboles, cobertura del dosel y la cobertura de hojarasca (Suazo-Ortuño et al., 2015). Estos cambios en los atributos del hábitat pueden tener un efecto importante en la abundancia y distribución de las especies, en particular en aquellas especies que tienen asociación estrecha con microhábitats particulares, como es el caso de los anfibios (Vitt, Ávila-Pires, Esposito, Sartorius \& Zani, 2007). De esta manera, el microhábitat se puede considerar como un factor importante en la distribución de las especies o de sus diferentes estadios de desarrollo (e.g. crías, juveniles y adultos) ya que la mayoría dependen de alguna estructura específica del hábitat en el cual viven 
(MacFadden \& Capen, 2002; Vitt et al., 2007; Bustos-Zagal, Manjarres \& Franco, 2013). Por lo tanto, identificar las características del microhábitat que permitan la supervivencia de las especies una vez que los bosques son perturbados, es esencial para realizar acciones de conservación tanto a nivel de especie como a nivel de comunidad. En el presente estudio evaluamos cambios en el uso del microhábitat de dos especies sintópicas de ranas arborícolas, Agalychnis dacnicolor y Smilisca fodiens a lo largo de un gradiente sucesional del BTS. Adicionalmente, evaluamos diferencias en el uso del microhábitat entre los estadios de desarrollo (crías, juveniles y hembras y machos adultos) y entre la estación del año (lluvias y secas). Nuestra hipótesis es que debido a que estas dos especies son generalistas del hábitat (SuazoOrtuño et al., 2015) tendrán como estrategia el cambio en el uso del microhábitat en respuesta a la sucesión secundaria del BTS y la temporada climática. Asimismo, se prevé que los cambios en las variables ambientales y estructurales del hábitat influenciarán la presencia, condición (biomasa y talla) y temperatura de los individuos.

\section{MATERIALES Y MÉTODOS}

Sitio de estudio: El área de estudio se ubica en la reserva de la biosfera ChamelaCuixmala y sitios aledaños localizados en la costa de Jalisco, México (19³0' N - 105 $03^{\circ}$ ' W). La temperatura anual en la región es de $25.1{ }^{\circ} \mathrm{C}$ y la precipitación promedio anual es de $788 \mathrm{~mm}$. La vegetación predominante es el bosque tropical seco (BTS), con una altura del dosel que va de los 5 a los $10 \mathrm{~m}$. La temporada de lluvias se extiende de julio a octubre y proporciona el $80 \%$ de la precipitación anual (Lott \& Atkinson, 2002). Las actividades humanas, particularmente desde 1960, han transformado el paisaje de la región en un mosaico complejo con distintas coberturas y usos del suelo, predominando los pastizales para uso de ganado y cultivos agrícolas, mezclado con arbustos y árboles aislados, lo que resta de la vegetación se compone de parches de bosque maduro y vegetación secundaria en diferentes etapas de sucesión. Las primeras etapas del proceso de sucesión son generalmente dominadas por hierbas y arbustos. Aproximadamente, 25 - $45 \%$ del paisaje en la región corresponde a la vegetación en diferentes etapas de sucesión y 55 $75 \%$ a bosque maduro (Burgos \& Mass, 2004).

Sistema de estudio: Los sitios de muestreo se ubicaron dentro de las parcelas que forman parte del proyecto "Manejo de bosques tropicales en la reserva de Chamela, Jalisco" (MABOTRO) (Fig. 1). Se seleccionaron 15 sitios de muestreo inmersos en un paisaje antropizado, integrado por pastizales con arbustos dispersos, árboles aislados, campos de cultivo y bosques secundarios en diferentes estadios de sucesión secundaria del bosque, los cuales se agruparon en cinco estadios sucesionales de vegetación de acuerdo con la edad de regeneración del bosque: pastizal, bosque inicial (5-6 años sin actividades agropecuarias), bosque joven (8-10 años sin actividades agropecuarias), bosque intermedio (15 a 17 años sin actividades agropecuarias) y bosque maduro (sin evidente signo de disturbio humano) (Cuadro 1). La edad de abandono de cada uno de los estadios sucesionales fue obtenida a través de entrevistas a los dueños de las tierras y por las características biofísicas (Trilleras, 2008). Se ubicaron tres sitios de muestreo por cada estadio sucesional y en cada sitio se ubicó una parcela permanente de $100 \times 100 \mathrm{~m}$, en la cual se llevó a cabo la búsqueda de individuos de Smilisca fodiens y Agalychnis dacnicolor y se evaluó el uso del microhábitat.

Muestreo del microhábitat: Se realizaron ocho salidas al campo de abril 2012 a septiembre 2014. Cinco salidas se llevaron a cabo en periodo de lluvias (julio-octubre) y tres en periodo de secas (noviembre-junio). Los muestreos se realizaron durante la noche (21:00-04:00 h.) por cinco personas. Para la búsqueda de los ejemplares, se usó la técnica de Búsqueda por Detección Visual [Visual Encounter Surveys (Crump \& Scott, 1994)]. Una vez que un ejemplar era localizado, se procedió 


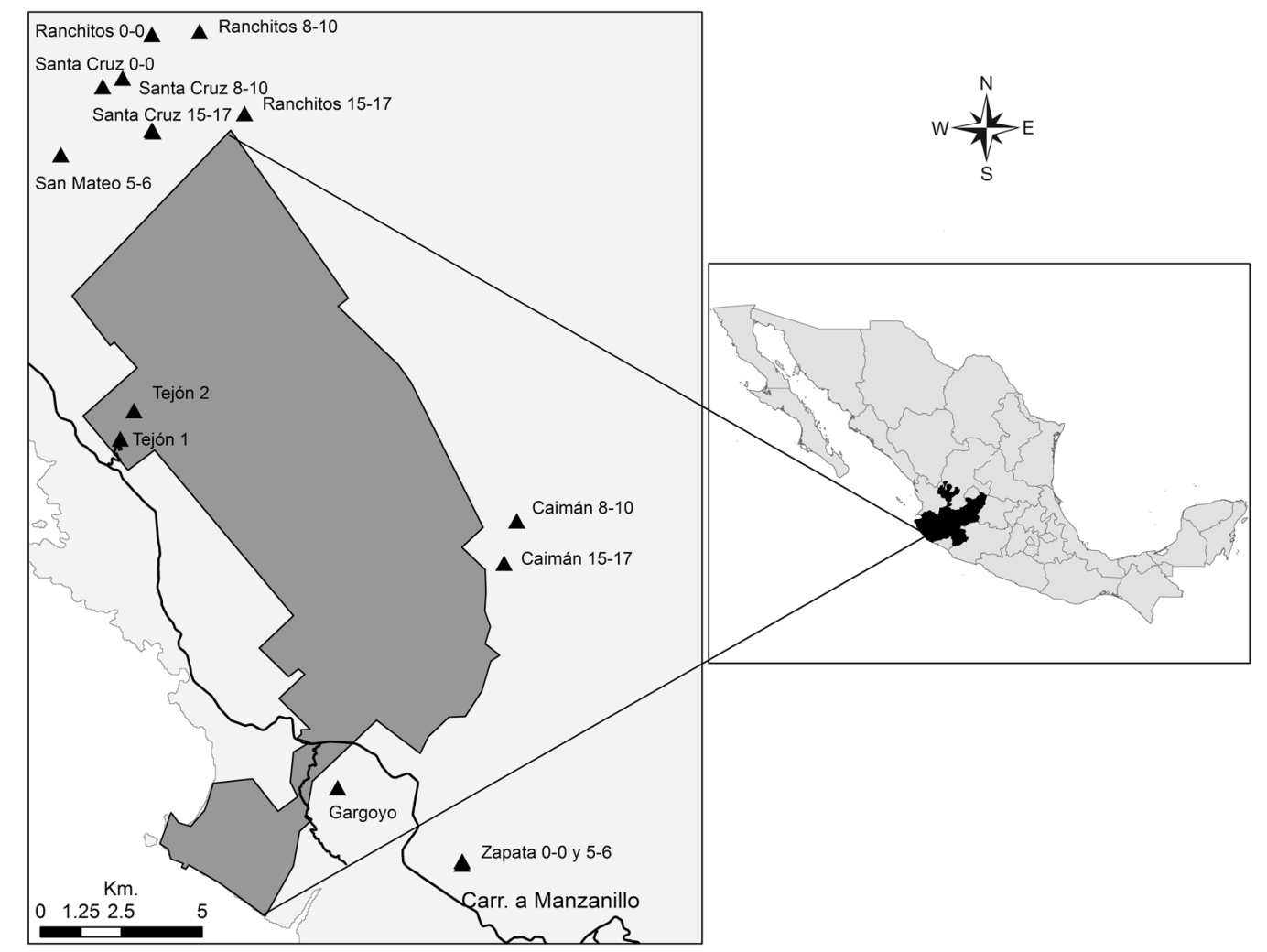

Fig. 1. Ubicación de los 15 sitios de muestreo en la Reserva de la Biósfera Chamela-Cuixlama y áreas aledañas, Jalisco, México.

Fig. 1. Location of the 15 sampling sites in the Biosphere Reserve Chamela-Cuixmala and adjacents areas, Jalisco, Mexico.

a su captura, registrando el sustrato donde se observó por primera vez (ramas de arbustos, ramas de árboles, sobre pasto seco, herbáceas, rocas, ramas y troncos secos sobre el suelo), además de la temperatura y humedad relativa del sustrato. De cada ejemplar capturado se midió la longitud hocico-cloaca (LHC) con un vernier, el peso $(\mathrm{g})$ con balanzas de precisión de diferente gramaje (marca Pesola) y la temperatura cloacal $\left({ }^{\circ} \mathrm{C}\right)$ con un termómetro de mercurio. Para evadir contar el mismo individuo durante el estudio, los animales fueron marcados con un corte de falange del tercer dedo. Además, se midió la temperatura y la humedad del ambiente a $150 \mathrm{~cm}$ del suelo con una microestación climática marca Kestrel 4000. Se estableció una parcela de $1 \mathrm{~m}^{2}$ alrededor del sitio de observación de cada individuo y se midió el porcentaje de cobertura de rocas, hojarasca, herbáceas, arbustos, árboles, suelo desnudo, ramas secas y troncos caídos. Adicionalmente, si el individuo se encontraba perchando en un árbol, se midió el diámetro a la altura del pecho (DAP) del árbol, la altura del refugio o percha y la altura del árbol o arbusto.

Se evaluaron los cambios en la biomasa (peso en gramos), LHC y temperatura corporal de los individuos de las dos especies entre estadios sucesionales y temporadas del año. Esto se realizó mediante un análisis de varianza multivariada basada en permutación (PERMANOVA). El análisis se basó en una matriz de similitud de Bray-Curtis, una prueba de Fisher con una suma de cuadrados secuencial tipo I y 9999 permutaciones. El diseño experimental tuvo cuatro factores: temporada 
CUADRO 1

Ubicación geográfica de los 15 sitios de muestreo con diferentes estadios sucesionales del Bosque Tropical Seco de Chamela, Jalisco, México

TABLE 1

Geographic location of the 15 sampling sites with different successional stages of Dry Tropical Forest in Chamela, Jalisco, México

\begin{tabular}{|c|c|c|c|c|c|}
\hline \multirow{2}{*}{ Sitio de estudio } & \multirow{2}{*}{$\begin{array}{l}\text { Acrónimos de los } \\
\text { sitios de estudio }\end{array}$} & \multirow{2}{*}{ Estadios Sucesionales } & \multicolumn{2}{|c|}{ Ubicación Geográfica } & \multirow{2}{*}{ Región } \\
\hline & & & Lat $\mathrm{N}$ & Long W & \\
\hline Ranchitos & R $0-0$ & Pastizal & $19^{\circ} 36^{\prime} 51.12^{\prime \prime}$ & $105^{\circ} 1^{\prime} 17.62^{\prime \prime}$ & 1 \\
\hline Santa Cruz & SC $0-0$ & Pastizal & $19^{\circ} 35^{\prime} 15.14 \prime$ & $105^{\circ} 02^{\prime} 5.05^{\prime \prime}$ & 1 \\
\hline Zapata & $\mathrm{Z} 0-0$ & Pastizal & $19^{\circ} 23^{\prime} 00^{\prime \prime}$ & $104^{\circ} 56^{\prime} 54.4^{\prime \prime}$ & 3 \\
\hline Santa Cruz & SC 5-6 & Bosque inicial & $19^{\circ} 35^{\prime} 13.62 ”$ & $105^{\circ} 02^{\prime} 4.03^{\prime \prime}$ & 1 \\
\hline Zapata & Z 5-6 & Bosque inicial & $19^{\circ} 23 ’ 2.8^{\prime \prime}$ & $104^{\circ} 56^{\prime} 54.42^{\prime \prime}$ & 3 \\
\hline San Mateo & SM 5-6 & Bosque inicial & 19॰34'50.64’' & $105^{\circ} 03^{\prime} 36.37^{\prime \prime}$ & 2 \\
\hline Ranchitos & R $8-10$ & Bosque joven & $19^{\circ} 36^{\prime} 53.92^{\prime \prime}$ & $105^{\circ} 01^{\prime} 17.45^{\prime \prime}$ & 1 \\
\hline Caimán & C $8-10$ & Bosque joven & $19^{\circ} 28^{\prime} 43.61^{\prime \prime}$ & $104^{\circ} 55^{\prime} 59.69^{\prime \prime}$ & 2 \\
\hline Santa Cruz & SC $8-10$ & Bosque joven & $19^{\circ} 36^{\prime} 7.33^{\prime \prime}$ & $105^{\circ} 02^{\prime} 34.54^{\prime \prime}$ & 1 \\
\hline Ranchitos & R $15-17$ & Bosque intermedio & $19^{\circ} 35^{\prime} 31.77^{\prime \prime}$ & $105^{\circ} 00^{\prime} 32.24^{\prime \prime}$ & 1 \\
\hline Santa Cruz & SC $15-17$ & Bosque intermedio & $19^{\circ} 35^{\prime} 58.8^{\prime \prime}$ & $105^{\circ} 02^{\prime} 54.5^{\prime \prime}$ & 1 \\
\hline Caimán & C $15-17$ & Bosque intermedio & $19^{\circ} 28^{\prime} 1.3^{\prime \prime}$ & $104^{\circ} 56^{\prime} 12.42^{\prime \prime}$ & 2 \\
\hline Gargoyo & G & Bosque maduro & 19²4’16.4”' & $104^{\circ} 58^{\prime} 59.0^{\prime \prime}$ & 2 \\
\hline Tejón 1 & $\mathrm{~T} 1$ & Bosque maduro & 19³0’05.9”' & $105^{\circ} 02^{\prime} 36.9^{\prime \prime}$ & 2 \\
\hline Tejón 2 & $\mathrm{~T} 2$ & Bosque maduro & $19^{\circ} 30^{\prime} 34.1 ”$ & $105^{\circ} 02^{\prime} 23.3^{\prime \prime}$ & 2 \\
\hline
\end{tabular}

Las regiones agrupan a los sitios cercanos entre sí.

Regions arrange sites near each other.

(fijo) con dos niveles (lluvias y secas); salida al campo con ocho niveles anidado en temporada (aleatorio), estadio de sucesión (fijo) con cinco niveles (pastizal, bosque inicial, bosque joven, bosque intermedio y bosque maduro), y sitio con tres niveles anidados en los estadios (aleatorio). Para asignar a un individuo en la categoría de estadio de desarrollo de cría, para A. dacnicolor fueron aquellos individuos con menos de $30 \mathrm{~mm}$ LHC, y menos de $25 \mathrm{~mm}$ para $S$. fodiens; para la fase juvenil, a aquellos individuos con LHC de 31 a $55 \mathrm{~mm}$ LHC para A. dacnicolor y de 26 a $50 \mathrm{~mm}$ para $S$. fodiens; en el caso de adultos, para $A$. dacnicolor fueron los individuos mayores a $55 \mathrm{~mm}$ LHC, y los mayores a $51 \mathrm{~mm}$ para $S$. fodiens. Todos los análisis se realizaron con el factor región como covariable (con tres niveles, región 1 Ranchitos-Santa Cruz, región 2 Bosques MaduroCaimanes, y Región 3 Zapata; Cuadro 1), bajo el supuesto que la cercanía geográfica de los sitios de muestreo podría tener un efecto. Cuando se encontraron efectos en algún factor o sus interacciones, se realizó una comparación a posteriori por pares con el estadístico $t$. Los análisis fueron realizados con el programa PRIMER v7 y PERMANOVA add on (Clarke \& Gorley, 2015).

Se realizó una ordenación estadística de los sitios a partir de distancias euclidianas en un análisis de componentes principales (PCA). Se determinó la correlación de Pearson de las variables con los ejes de ordenación. Previo al PCA, se utilizó el coeficiente de correlación de Pearson para determinar la correlación lineal entre las variables ambientales y estructurales del microhábitat y así poder excluir las variables colineales (correlación superior al $80 \%$ ) en análisis posteriores. Se usó un Análisis de Componentes Principales (PCA) para identificar la asociación de $A$. dacnicolor y $S$. fodiens con los atributos del hábitat en cada 
uno de los estadios sucesionales del BTS. Posteriormente, se utilizó un análisis de correlación de Pearson para identificar los atributos del hábitat significativamente asociados con cada uno de los dos Componentes Principales. Para estimar diferencias en el uso del microhábitat de ambas especies entre los diferentes estadios sucesionales, se realizó un ANOVA de una vía para cada uno de los componentes principales (CP) (con eigenvalor $>1$ ) derivados del PCA antes mencionado, los cuales fueron utilizados como las nuevas variables dependientes. Este método se ha empleado para reducir la dimensionalidad de variables y comparar entre poblaciones (Zúñiga-Vega, Suárez-Rodríguez, Espinoza-Pérez \& Johnson, 2011).

A partir de modelos lineales basados en distancias (rutina DistLM) se identificaron las variables ambientales que mejor explicaron los cambios en la biomasa, talla y temperatura corporal de los individuos de las dos especies. Los mejores modelos se evaluaron a partir del criterio de información de Akaike para muestras pequeñas (AICc) y el valor de $\mathrm{r}^{2}$ ajustado. Los análisis fueron realizados con el programa PRIMER v7 y PERMANOVA add on (Clarke \& Gorley, 2015).

Para caracterizar el uso del microhábitat que utilizaron ambas especies en sus distintas etapas de desarrollo en los diferentes estadios sucesionales se utilizó un análisis de coordenadas principales (PCoA), el cual está basado en una matriz de similitud de Bray-Curtis.

\section{RESULTADOS}

Abundancia de las especies: En total se registraron 58 individuos de Agalychnis dacnicolor y 67 de Smilisca fodiens (Cuadro 2). A. dacnicolor no presentó diferencias en abundancia entre salidas al campo, sitios de muestreo, estadios sucesionales o temporada climática. En S. fodiens la abundancia varió entre salidas, temporada climática y estadio sucesional $(F=2.319$; p-perm $=0.011)$. Durante la temporada de secas la abundancia de $S$. fodiens fue mayor en el bosque intermedio $(\mathrm{t}=11728 ; \mathrm{P}(\mathrm{M})=0$ 042), mientras que durante la época de lluvias fue mayor en el pastizal $(\mathrm{t}=26091 ; \mathrm{P}(\mathrm{M})=0$ 001).

Cambios en biomasa, talla y temperatura corporal: No hubo diferencias significativas en la biomasa de $A$. dacnicolor entre estadios sucesionales ni entre temporadas climáticas, pero sí entre los sitios de muestreo $(\mathrm{F}=3.9377$; p-perm $=0.0315)$. En A. dacnicolor LHC varió significativamente entre las salidas de campo, estadios sucesionales y temporada climática ( $\mathrm{F}$ $=33.475$; p-perm $=0.0001$ ); durante la temporada de secas LHC no varió entre los estadios

\section{CUADRO 2}

Número de individuos muestreados de Agalychnis dacnicolor y Smilisca fodiens en cada uno de los estadios sucesionales por época del año en la región de Chamela, Jalisco, México

TABLE 2

Number of sampled individuals of Agalychnis dacnicolor and Smilisca baudini in each of the successional stages by climatic season in the region of Chamela, Jalisco, México

\begin{tabular}{|c|c|c|c|c|c|c|}
\hline & Pastizal & Bosque inicial & Bosque joven & Bosque intermedio & Bosque maduro & Total \\
\hline \multicolumn{7}{|c|}{ Agalychnis dacnicolor } \\
\hline Lluvias & 9 & 5 & 6 & 0 & 3 & 23 \\
\hline Secas & 6 & 4 & 10 & 0 & 15 & 35 \\
\hline Total & 15 & 9 & 16 & 0 & 18 & 58 \\
\hline \multicolumn{7}{|c|}{ Smilisca fodiens } \\
\hline Lluvias & 1 & 7 & 5 & 8 & 5 & 26 \\
\hline Secas & 16 & 8 & 10 & 7 & 0 & 41 \\
\hline Total & 17 & 15 & 15 & 15 & 5 & 67 \\
\hline
\end{tabular}


sucesionales; pero durante la temporada de lluvias LHC fue diferente en el bosque joven, en el que se presentaron mayores tallas en la salida 4 respecto a la salida $6(\mathrm{t}=3.0267 ; \mathrm{p}=$ $0.0286)$ y $8(\mathrm{t}=5.1491$ y $\mathrm{p}=0$ 0383). En contraste, la biomasa y LHC de $S$. fodiens no varió entre sitios, estadios sucesionales, temporada climática o salidas al campo (Cuadro 3).

La temperatura corporal de A. dacnicolor varió entre estadios sucesionales $(\mathrm{F}=$ 5.3991 ; p-perm $=0.0188)$ y entre temporadas climáticas $(\mathrm{F}=3.6689 ;$ p-perm $=0.0215)$. Los promedios de las temperaturas corporales fueron mayores en el bosque maduro, seguido del bosque inicial, pastizal y bosque joven. Durante la temporada de lluvias las temperaturas corporales fueron similares entre los estadios sucesionales, mientras que en la temporada de secas, las pruebas a posteriori indicaron diferencias significativas entre el bosque inicial y el bosque joven $(\mathrm{t}=3.3653$; p-perm $=0.001)$. Adicionalmente, se observó que la variabilidad en la temperatura corporal (expresada por las distancias euclidianas) en la temporada de secas, se redujo al incrementar la edad del estadio sucesional [pastizales (4.4444); bosque temprano (2.4); bosque joven (1.9333); bosque maduro (1.3333)]. A pesar de que no hubo diferencias en la época de lluvias en las temperaturas corporales, la variabilidad fue menor en bosques maduro e intermedio [bosque joven (4.1333), pastizales (3.2), bosque inicial (3.1667); bosque maduro (1.1333), bosque intermedio (0)].

La temperatura corporal de $S$. fodiens varió significativamente entre temporadas climáticas y estadios sucesionales $(\mathrm{F}=55.045$; p-perm $=0.0294$ ). Durante la temporada de lluvias se observaron diferencias significativas entre el pastizal y el bosque intermedio $(\mathrm{t}=3.0545$; $\mathrm{P}(\mathrm{MC})=0.0073)$. También durante esta temporada se registró una reducción en la variación de la temperatura corporal de $S$. fodiens expresada por las distancias euclidianas entre los estadios sucesionales, siendo mayor en el pastizal (0.27331), seguido por el bosque joven (0.1733), el bosque intermedio (0.14054) y el bosque inicial (0.13852). Durante la temporada
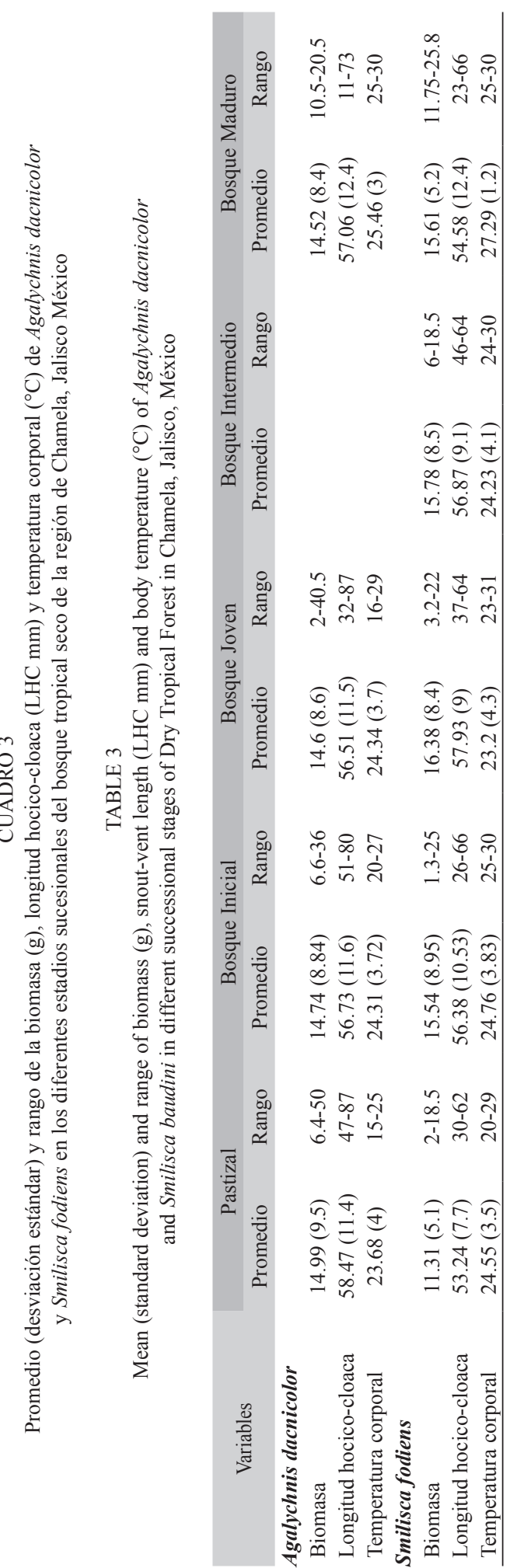
de secas la temperatura corporal de $S$. fodiens varió significativamente entre el pastizal y el boque inicial $(\mathrm{t}=4.3186$; $\mathrm{P}(\mathrm{MC})=0.0182)$, pastizal y bosque intermedio $(\mathrm{t}=3.2541 ; \mathrm{P}$ $(\mathrm{MC})=0.0155)$, bosque inicial y bosque intermedio $(\mathrm{t}=4.0084$; $\mathrm{p}$-perm $=0.0176)$ y bosque intermedio y bosque joven $(\mathrm{t}=19.223$; $\mathrm{P}(\mathrm{MC})$ $=0.0329)$. En esta temporada, la variación en la temperatura corporal de $S$. fodiens expresada por las distancias euclidianas fue menor en pastizales (0) y los bosques intermedios (0.087275), y se incrementó en el bosque inicial (0.16609), bosque maduro (0.21032) y el bosque joven (0.2253).

Hábitat de las especies: De las 15 variables ambientales y estructurales que fueron medidas, solo la relación entre la temperatura del sustrato y la temperatura ambiental, así como la relación de la humedad del sustrato y la humedad ambiental presentaron colinearidad $\left(\mathrm{r}^{2}>83 \%\right.$; Apéndice 1 y 2$)$, por lo que para el PCA se eliminaron las variables de temperatura y humedad ambiental. Los dos primeros componentes principales (PC) explicaron el $50.7 \%$ del total de la variación ambiental entre los sitios de muestreo. El PC1 explicó el $32.5 \%$, siendo las variables de mayor peso la temperatura del sustrato, altura del refugio, altura del árbol o arbusto y el porcentaje de árboles. Por su parte, el PC2 explicó el $18.2 \%$ de la variación ambiental, siendo las variables de mayor peso el porcentaje de arbusto, suelo desnudo, herbáceas, ramas secas y troncos caídos (Fig. 2).

Los primeros $6 \mathrm{PC}$ presentaron un eigenvalor mayor de uno. Los ANOVAs de una vía indicaron diferencias significativas para el PC1 entre estadios $\left(\mathrm{F}_{4}=12.87, \mathrm{p}<0.001\right)$ y entre especies $\left(\mathrm{F}_{1}=4.99, \mathrm{p}<0.001\right)$, siendo las variables de mayor peso la altura del refugio $(0.20)$ y el porcentaje de árbol $(0.23)$;

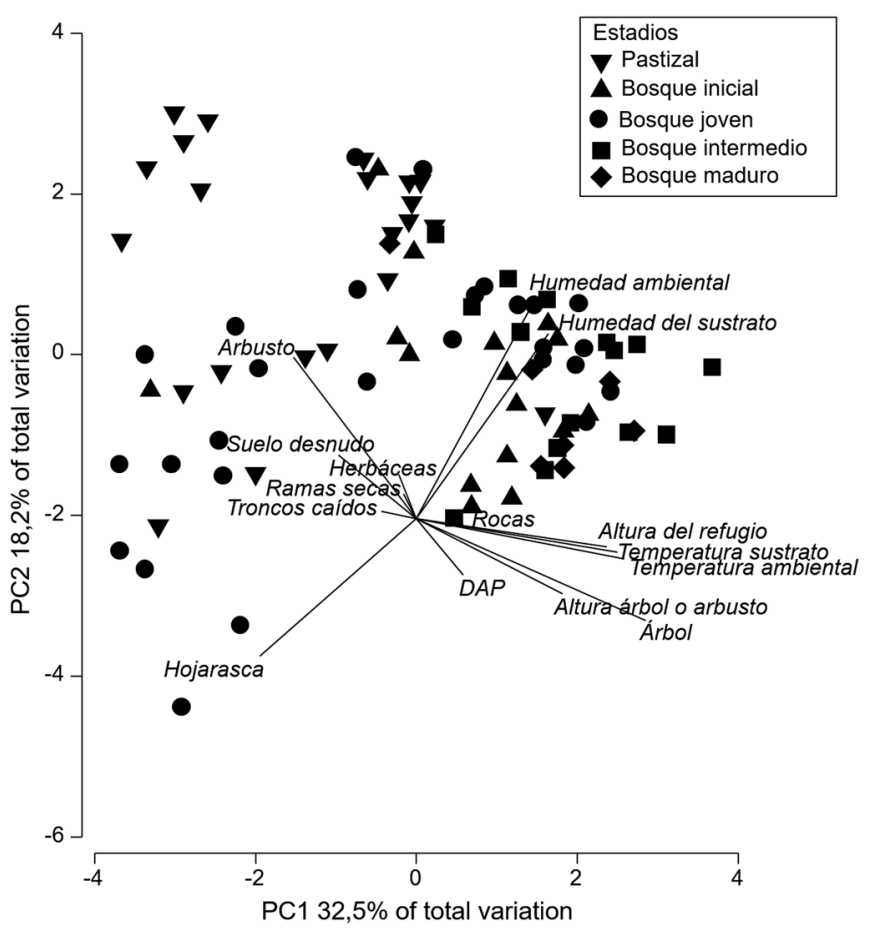

Fig. 2. Biplot del PCA representando las distintas variables ambientales en relación a los estadios sucesionales del bosque tropical seco de la región de Chamela, Jalisco, México.

Fig. 2. PCA biplot representing the different environmental variables in relation to successional stages of the dry tropical forest in the region of Chamela, Jalisco, Mexico. 
para el PC3 entre estadios $\left(\mathrm{F}_{4}=5.79, \mathrm{p}<0.001\right)$ las variables de mayor peso fueron la humedad del sustrato (0.20) y el DAP (0.20); para el PC4 hubo diferencias entre estadios $\left(\mathrm{F}_{4}=3.10, \mathrm{p}=\right.$ 0.01 ), siendo la variable de mayor peso el porcentaje de pasto (0.33); por último encontramos diferencias para el PC5 $\left(\mathrm{F}_{4}=3.12, \mathrm{p}<0.0001\right)$, siendo la variable de mayor peso el porcentaje de herbáceas (0.50). Las pruebas a posteriori indicaron que el microhábitat utilizado por ambas especies difiere en el bosque joven en el PC1 $(\mathrm{p}<0.001)$ y en el PC3 $(\mathrm{p}=0.011)$; en $A$. dacnicolor los estadios sucesionales difirieron en el PC1 entre el bosque joven y el bosque maduro $(\mathrm{p}=0.041)$ y entre el pastizal y el bosque maduro $(p=0.004)$, en el PC3 difirieron el bosque joven y el pastizal $(p=0.003)$, en el PC4 difirieron el bosque joven y el bosque inicial $(p=0.003)$ y en el PC5 difirieron el bosque joven y el pastizal $(\mathrm{p}=0.003)$. En $S$. fodiens los estadios sucesionales difirieron en el PC1 el pastizal con el bosque inicial $(p=0.005)$, bosque joven $(\mathrm{p}=0.007)$, bosque intermedio ( $\mathrm{p}$ $<0.001)$ y bosque maduro $(\mathrm{p}=0.001)$.

En la asociación de $A$. dacnicolor y $S$. fodiens con los atributos del hábitat en cada uno de los estadios sucesionales, el PCA mostró que en el pastizal el PC1 explicó el $21.1 \%$ de la variación, mientras que el PC2 el $18.8 \%$ de la variación; las variables que separaron a $A$. dacnicolor y $S$. fodiens en este estadio fueron la altura del árbol o arbusto, el porcentaje de herbáceas y el número de estratos (Fig. 3A). En el bosque inicial el PC1 explicó el $27.7 \%$ de la variación total y el PC2 explicó el $24.4 \%$; las variables que significativamente separaron a las especies fueron la temperatura del sustrato, humedad del sustrato, porcentaje de ramas secas, porcentaje de herbáceas, porcentaje de árboles y la altura del refugio (Fig. 3B). En el bosque joven el PC1 explicó el $29.6 \%$ de la variación, mientras que el PC2 explicó el 17.1 $\%$; las variables que separaron a las especies fueron la temperatura del sustrato, humedad del sustrato, porcentaje de árboles, porcentaje de hojarasca, el diámetro a la altura del pecho y el porcentaje de troncos caídos (Fig. 3C). En el bosque intermedio, el PC1 explicó el $37.6 \%$ de la variación y el PC2 explicó el 18.9 \%; las variables que separaron a las especies en este estadio fueron el diámetro a la altura del pecho, porcentaje de hojarasca, el porcentaje de troncos caídos, porcentaje de árboles, porcentaje de herbáceas y porcentaje de ramas secas (Fig. 3D). Para el bosque maduro el PC1 explicó el $27.8 \%$ de la variación y el PC2 el $23.5 \%$; para este estadio las variables de mayor peso que separaron a ambas especies fueron la temperatura del sustrato, diámetro a la altura del pecho, porcentaje de hojarasca, porcentaje de troncos caídos, porcentaje de árboles, porcentaje de herbáceas y porcentaje de ramas secas (Fig. 3E).

Relación entre biomasa, LHC y temperatura corporal con las variables ambientales: En $A$. dacnicolor la biomasa estuvo mejor explicada por las herbáceas $\left(\mathrm{R}^{2}\right.$ ajustada $\left.=0.20 \%\right)$, seguido por la interacción de las herbáceas con los troncos caídos $\left(\mathrm{R}^{2}\right.$ ajustada $=0.30 \%)$ y por la interacción entre la hojarasca, herbáceas y troncos caídos $\left(\mathrm{R}^{2}\right.$ ajustada $=0.35 \%$ ). Mientras que para LHC la variable más explicativa fue la altura del árbol o arbusto $\left(\mathrm{R}^{2}\right.$ ajustada $\left.=0.42 \%\right)$, seguido por la interacción de DAP con la altura del árbol o arbusto $\left(\mathrm{R}^{2}\right.$ ajustada $\left.=0.52 \%\right)$ y por la interacción entre DAP, la altura del árbol o arbusto y herbáceas $\left(\mathrm{R}^{2}\right.$ ajustada $\left.=0.60 \%\right)$. En cuanto a temperatura corporal, la mejor variable que la explicó fue la temperatura del sustrato $\left(\mathrm{R}^{2}\right.$ ajustada $=0.67 \%$ ), seguido por la interacción de la temperatura del sustrato con herbáceas $\left(\mathrm{R}^{2}\right.$ ajustada $\left.=0.74 \%\right)$, y por la interacción entre temperatura del sustrato, la hojarasca y el arbusto $\left(\mathrm{R}^{2}\right.$ ajustada $=0.80 \%$ ) (Apéndice 3 ).

Las variables que mejor explicaron la biomasa de $S$. fodiens fueron las ramas secas $\left(\mathrm{R}^{2}\right.$ ajustada $=0.11 \%$ ), seguida por la interacción de ramas secas con la humedad del sustrato $\left(\mathrm{R}^{2}\right.$ ajustada $\left.=0.15 \%\right)$ y por la interacción entre humedad del sustrato, hojarasca y ramas secas $\left(\mathrm{R}^{2}\right.$ ajustada $=0.17 \%$ ) (Apéndice 3$)$. La variable que mejor explicó LHC en esta especie fue ramas secas $\left(\mathrm{R}^{2}\right.$ ajustada $\left.=0.03 \%\right)$, seguida por la interacción de la altura del refugio 
(A)

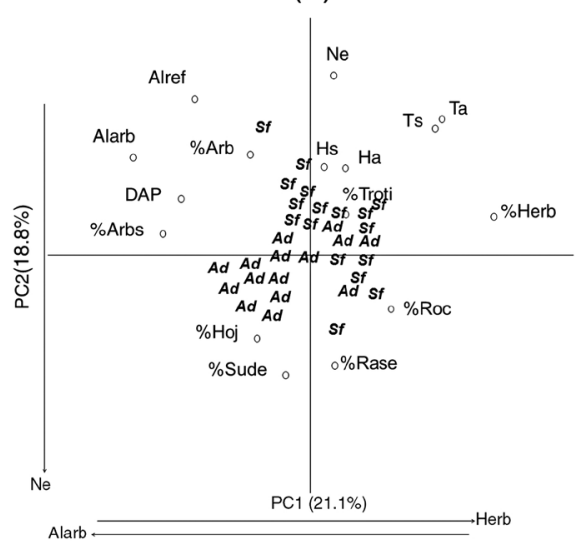

(C)

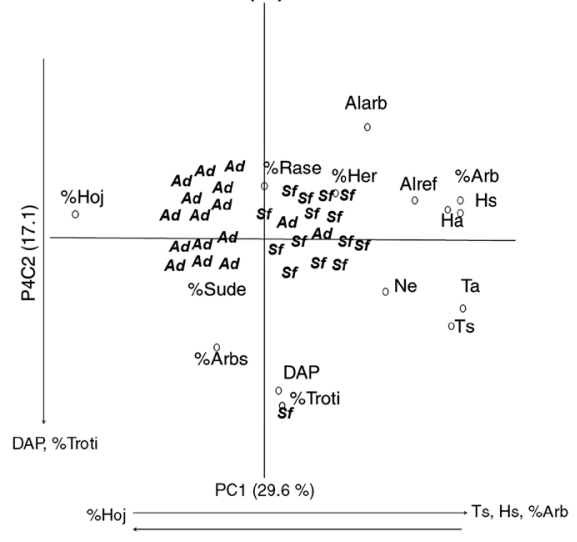

(B)

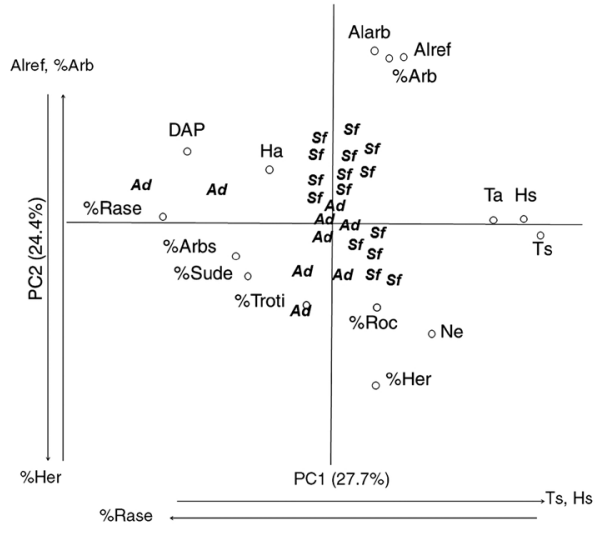

(D)

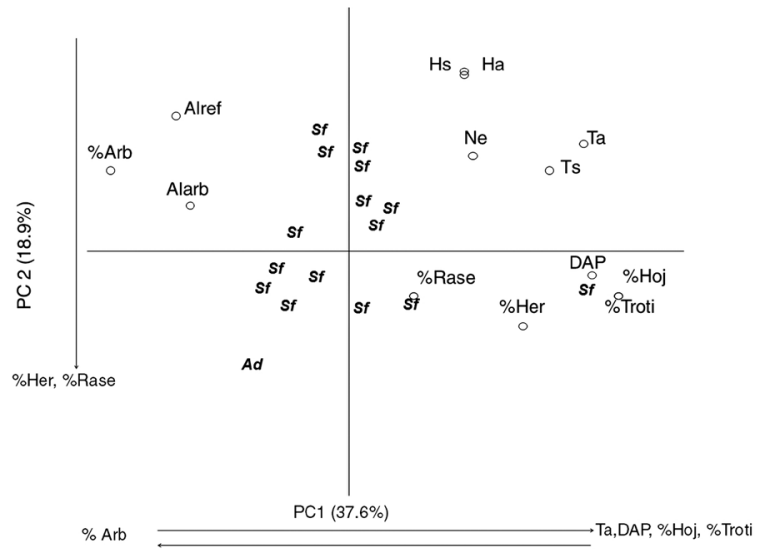

(E)

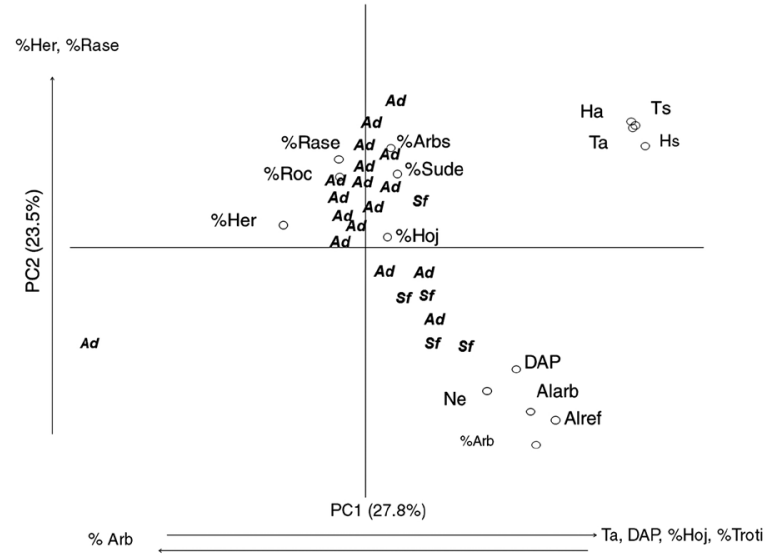

Fig. 3. PCA de la distribución en uso del microhábitat de Agalychnis dacnicolor y Smilisca fodiens en los diferentes estadios sucesionales: A) Pastizales, B) Bosque Temprano, C) Bosque Joven, D) Bosque Intermedio, y E) Bosque Maduro.

Fig. 3. PCA of distribution of microhabitat use by Agalychnis dacnicolor and Smilisca fodiens in the different successional stages: A) Pastures, B) Early Forest, C) Young Forest, D) Intermediate Forest, and E) Mature Forest. 
con las ramas secas $\left(\mathrm{R}^{2}\right.$ ajustada $\left.=0.06 \%\right)$ y por la interacción entre la altura del refugio, rocas y ramas secas $\left(\mathrm{R}^{2}\right.$ ajustada $\left.=0.06 \%\right)$. $\mathrm{La}$ temperatura corporal de esta especie fue explicada por la temperatura del sustrato $\left(\mathrm{R}^{2}\right.$ ajusta$\mathrm{da}=0.12 \%$ ), seguida por la interacción de la temperatura del sustrato con las herbáceas $\left(\mathrm{R}^{2}\right.$ ajustada $=0.20 \%$ ), y por la interacción entre la temperatura del sustrato, las herbáceas y ramas secas $\left(\mathrm{R}^{2}\right.$ ajustada $\left.=0.21 \%\right)($ Apéndice 3$)$.

\section{Uso del microhábitat entre estadios de} desarrollo: Se registró diferencia en el uso del microhábitat entre los estadios de desarrollo de A. dacnicolor [crías $(\mathrm{N}=6)$, juveniles $(\mathrm{N}=16)$ y machos $(\mathrm{N}=34)$ y hembras $(\mathrm{N}=16)$ adultos] entre los diferentes estadios sucesionales $(\mathrm{F}=2.3472 ; \mathrm{p}$-perm $=0.0486)$, además de la interacción de estadio sucesional-estadio de desarrollo la cual fue significativa $(\mathrm{F}=2.3337$; p-perm $=0.0067)$ y la interacción temporada climática-estadio de desarrollo $(\mathrm{F}=3.8101$; p-perm $=0.0085)$. La prueba a posteriori indicó diferencias significativas entre la temporada climática en los machos $(\mathrm{t}=3.0581$; $\mathrm{p}$-perm $=$ $0.0024)$, mientras que para los demás estadios de desarrollo no hubo diferencias significativas entre temporadas climáticas. En cuanto a los estadios sucesionales, en hembras se registraron interacciones significativas entre el bosque inicial y el bosque joven $(\mathrm{t}=2.1263$; $\mathrm{p}$-perm $=0.0414) \mathrm{y}$ entre el bosque maduro y el bosque joven $(\mathrm{t}=2.1263$; p-perm $=0.0462)$. Para machos, se registraron interacciones significativas entre el bosque inicial y el bosque maduro $(t=2.2924$; p-perm $=0.0291)$, entre el bosque intermedio y el bosque maduro $(\mathrm{t}=2.8508$; p-perm $=0.0055)$, entre el bosque maduro y el pastizal $(\mathrm{t}=2.8508$; $\mathrm{p}$-perm $=0.0069)$ y entre el bosque maduro y el bosque joven $(\mathrm{t}=2.1607$; p-perm $=0.0376$ ). En juveniles y crías no hubo diferencia significativa en sus interacciones.

El PCoA para los estadios de desarrollo y los estadios sucesionales mostró que los machos de A. dacnicolor están mayormente asociados a los bosques maduros, mientras que los demás estadios de desarrollo (hembras, crías, juveniles) están mayormente asociados a los estadios de pastizal y bosque joven (Fig. 4A). Los machos $(\mathrm{N}=18)$ de $S$. fodiens estuvieron mayormente asociados al bosque intermedio, bosque joven y bosque inicial; mientras que las hembras $(\mathrm{N}=29)$ estuvieron asociadas al bosque joven y bosque inicial; los juveniles $(\mathrm{N}=$ 18) estuvieron mayormente asociados al boque maduro, bosque joven y pastizal; y las crías ( $\mathrm{N}$ $=5$ ) al bosque joven (Fig. 4B).

\section{DISCUSIÓN}

La rana verde $A$. dacnicolor no presentó diferencias significativas en abundancia entre estadios sucesionales ni entre temporadas climáticas, mientras que la abundancia de la rana $S$. fodiens varió significativamente entre estadios sucesionales y entre temporadas climáticas. Aunque ambas especies se registraron en bosques secundarios y maduros, A. dacnicolor presentó asociación con el bosque maduro, mientras que $S$. fodiens presentó asociación con los lugares perturbados. A pesar de que se ha reportado que ambas especies son generalistas de hábitat y que poseen rasgos morfo-fisiológi$\cos$ que les permiten vivir en un amplio rango de condiciones ambientales (Suazo-Ortuño et al., 2015), nuestros resultados muestran que S. fodiens presenta mayor plasticidad ecológica que $A$. dacnicolor. La mayor abundancia registrada en ambas especies durante la temporada de lluvias, en comparación con la temporada de secas, puede estar asociada a sus hábitos. $\mathrm{Su}$ periodo de reproducción ocurre en los meses lluviosos y por lo tanto, los individuos son más activos y su detectabilidad aumenta (julio-octubre; Lott \& Atkinson, 2002). Por otro lado, se ha reportado que varias especies de anfibios presentan mayor abundancia en bosques fragmentados en respuesta al mayor número de cuerpos de agua (generalmente artificiales) y a la menor distancia entre ellos, que los encontrados en el bosque continuo (Joly, Miaud, Lehmann \& Grolet, 2001). En cuanto a biomasa, tanto A dacnicolor como $S$. fodiens no presentaron diferencias entre estadios sucesionales o entre temporadas climáticas. En relación con LHC, A. dacnicolor 

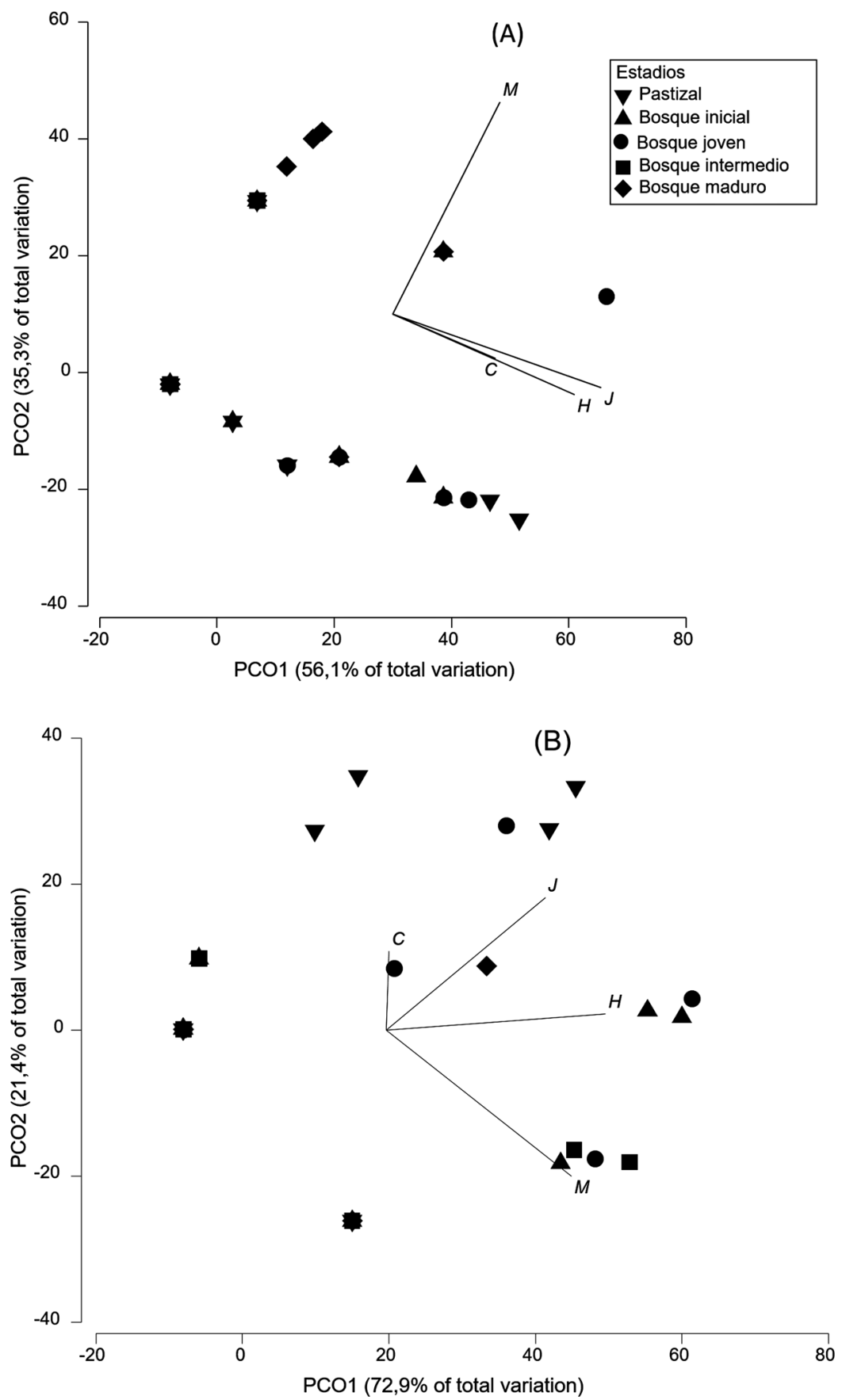

Fig. 4. PCoA que muestra la distribución de los diferentes estadios de desarrollo de (a) Agalychnis dacnicolor y (b) Smilisca fodiens en relación a los estadios sucesionales de la vegetación. $\mathrm{M}=$ machos, $\mathrm{H}=$ hembras, $\mathrm{J}=$ juveniles y $\mathrm{C}=$ crías.

Fig. 4. PCoA showing distibution of the different developmental stages of (a) Agalychnis dancicolor and (b) Smilisca fodiens in relation to vegetation succesional stages. $\mathrm{M}=$ males, $\mathrm{H}=$ females, $\mathrm{J}=$ juveniles and $\mathrm{C}=$ hatchlings. 
presentó mayores tallas durante la temporada de lluvia que durante la temporada de secas. Se ha reportado que tanto el tipo de hábitat como la temporada climática influyen en el peso y LHC de algunas especies de ranas arborícolas del género Phyllomedusa (Neckel-Oliveira \& Gascon, 2006). Uno de los factores que puede explicar las diferencias encontradas en la talla de A. dacnicolor es la disponibilidad de presas, ya que la estacionalidad que presenta el sitio de estudio altera la disponibilidad de alimento a lo largo del año (Bradford \& García-Aguayo, 1992). Se conoce que durante la época de lluvias diversas especies de anfibios consumen un mayor volumen de presas que en la temporada de secas, y en consecuencia incrementan su talla y peso (Cuevas \& Martori, 2007; Martínez-Coronel \& Pérez-Gutiérrez, 2011; Del Río-García, Serrano-Cardozo \& RamírezPinilla, 2014). Es posible, que el hecho de que $S$. fodiens no haya presentado diferencias en biomasa y LHC entre estadios sucesionales y temporada climática, se deba a que esta especie aún cuando presenta preferencia por el orden Lepidoptera y la familia Termitidae, tiene la capacidad de cambiar su dieta a lo largo de la sucesión secundaria del bosque (MezaSantiago, 2014), lo que le permitiría consumir diversas presas y alcanzar las mismas tallas en los diferentes estadios sucesionales del BTS.

La temperatura corporal de A. dacnicolor y $S$. fodiens varió entre estadios sucesionales y temporadas climáticas. Estas diferencias podrían estar asociadas en mayor medida a la estacionalidad del sitio y a las diferencias en la complejidad estructural de los diferentes estadios sucesionales (Suazo-Ortuño et al., 2015). Aún y cuando la temperatura corporal de las dos especies varió en respuesta a la sucesión secundaria del bosque y a la temporada climática, cada especie presentó una respuesta diferente. En el caso de A. dacnicolor, la temperatura promedio de los individuos fue significativamente mayor en el bosque maduro que en el resto de los estadios sucesionales, quizá como resultado de una menor intensidad de la circulación del aire, debido a la mayor complejidad estructural del bosque maduro (Promis, Caldentey
\& Ibarra, 2010), lo que podría favorecer el incremento de la temperatura. En la época de lluvias, por el contrario, no se encontraron diferencias entre los estadios sucesionales, quizá debido a que el follaje de la vegetación limita la circulación del aire. Por otro lado, es importante destacar que la variabilidad de la temperatura corporal entre los individuos de $A$. dacnicolor se redujo al incrementar el estadio de sucesión durante la temporada de secas y en el caso de $S$. fodiens la variación en la temperatura corporal fue menor en el pastizal y el bosque intermedio que en el resto de los estadios sucesionales. Así, el efecto que tienen los estadios sucesionales del bosque tropical seco y la interacción con las estaciones climáticas en la temperatura corporal de los individuos de ambas especies, puede tener diferentes implicaciones fisiológicas dependiendo del estadio de sucesión, ya que en organismos ectotérmicos como los anuros, la temperatura puede afectar el comportamiento y los procesos metabólicos, influyendo a su vez en el desarrollo de los individuos y su distribución en los diferentes ambientes (Toft, 1985; Webb \& Shine, 1998). La variación encontrada en las temperaturas corporales en ambas especies aunado a los resultados del PCoA sugiere que estas especies están siendo afectadas diferencialmente por las condiciones del hábitat, así como por la estructura de la vegetación presente en los diferentes estadios sucesionales del BTS. Los individuos de $S$. fodiens presentaron una temperatura más constante en los pastizales, mientras que los de A. dacnicolor en el bosque maduro.

Los resultados de la variación ambiental y estructural en los diferentes estadios sucesionales obtenida por el análisis de componentes principales (PCA) separó al bosque joven, bosque intermedio y bosque maduro del resto de los estadios sucesionales en función del incremento en la temperatura del sustrato, así como del incremento en la altura del refugio, altura del árbol o arbusto y del porcentaje de árboles, características estructurales que se incrementan conforme aumentan los años de regeneración del bosque. De acuerdo con Cortés-Gómez et al. (2013) es necesario una alta 
densidad de plantas leñosas $(>231$ individuos por $500 \mathrm{~m}^{2}$ ) para poder preservar una alta composición de especies de anfibios en bosques maduros. Adicionalmente, estas características proveen a las diversas especies de anfibios de condiciones óptimas en sus microhábitats para perchar, alimentarse, aparearse y anidar (Hilje \& Aide, 2012). Por otro lado, los bosques iniciales y pastizales se separaron del resto de los estadios sucesionales en función del incremento en el porcentaje de la cobertura de arbustos, suelo desnudo, herbáceas, ramas secas y troncos caídos, características propias de bosques con menores años de regeneración. Estas características incrementan la complejidad del hábitat produciendo una mayor gama de microhábitats con potencial de albergar una gran riqueza de anfibios (Rittenhouse, Harper, Rehard \& Semlitsch, 2008; Todd et al., 2008). Las variables ambientales y estructurales del hábitat que influyeron sobre la presencia de ambas especies dentro de los diferentes estadios sucesionales (temperatura del sustrato, altura del árbol o arbusto y los porcentajes de hojarasca, herbáceas, arbustos, árboles y suelo desnudo) concuerdan con lo reportado por Urbina-Cardona y Reynoso (2011) y Hillers, Veith y Rödel (2008), quienes detectaron que la altura sobre el suelo, humedad relativa, temperatura y la pendiente son las variables que influyen en la distribución de una alta cantidad de especies de anfibios. Las variables que mejor explicaron la biomasa, LHC y temperatura corporal de $S$. fodiens fueron las ramas secas y la temperatura del sustrato y en $A$. dacnicolor la biomasa estuvo mejor explicada por las herbáceas, la LHC por la altura del árbol o arbusto y la temperatura corporal por la temperatura del sustrato. Así, los diferentes elementos del microhábitat pueden influir marcadamente sobre los individuos, ya que por ejemplo, una capa gruesa de hojarasca puede preservar eficazmente la humedad en sitios fragmentados, amortiguando así las condiciones más secas y calientes (UrbinaCardona, Olivares-Pérez \& Reynoso, 2006; Harper et al., 2005). Sin embargo, las condiciones de los microhábitats de los anfibios pueden modificarse dependiendo de las condiciones climáticas, la orientación del borde y tamaño de los parches, lo cual influye fuertemente sobre la abundancia de los anfibios (Urbina-Cardona et al., 2006; Urbina-Cardona et al., 2012).

Los resultados de los PCA's y de los ANOVA's muestran que A. dacnicolor y $S$. fodiens utilizan de forma diferencial las variables ambientales y estructurales del microhábitat, conforme los estadios sucesionales aumentan en edad. La asociación de las especies con los atributos del hábitat sugiere que cada una de ellas está sujeta a requerimientos específicos en cada estadio sucesional. De acuerdo con Menin, Rossa-Feres y Giaretta (2005) la posición y tipo de sustratos utilizados por dos hílidos sintópicos (Hyla nana e Hyla sanborni) depende de la vegetación disponible en el hábitat, lo cual a su vez puede cambiar las dinámicas de la repartición de recursos entre especies que coexisten. En el presente estudio, también registramos diferencias en el microhábitat utilizado entre ambas especies sintópicas, siendo las variables que separaron a ambas especies, la altura del refugio y el porcentaje de árboles en los diferentes estadios sucesionales.

$A$. dacnicolor tiende a usar estos elementos de manera diferenciada en el bosque joven con respeto a los demás estadios sucesionales. De acuerdo a Hilje y Aide (2012), los bosques jóvenes proporcionan microhábitats adecuados para los anfibios ya que cuentan con un dosel cerrado, una alta humedad, y una capa profunda de hojarasca y abundantes artrópodos, lo que les provee de condiciones adecuadas para su persistencia. S. fodiens también presentó diferencias en el uso del microhábitat en todos los estadios sucesionales, sin embargo, esta diferencia solo estuvo dada por variaciones en la altura del refugio y por el porcentaje de árboles que está utiliza a lo largo de la sucesión.

A pesar de que conforme avanza la sucesión hay una mayor disponibilidad de hábitats para elegir, se pudo observar que $A$. dacnicolor mantiene una asociación con los estratos más bajos de la vegetación a lo largo de la sucesión, manteniendo un promedio de la altura de su refugio, por lo que nuestros resultados sugieren 
que $A$. dacnicolor es especialista de microhábitat, aun cuando ha sido reportada como especie generalista de hábitat al encontrarse en todos los estadios sucesionales del BTS (SuazoOrtuño et al., 2015). En este sentido, nuestros resultados destacan que aún cuando las especies se consideren generalistas de hábitat, algunas presentan especialización mientras que otras presentan mayor plasticidad en el uso del microhábitat. S. fodiens, quien también es considerada una especie generalista (Suazo-Ortuño et al., 2015), en este estudio mostró una amplia plasticidad en el uso del microhábitat al cambiar sus requerimientos a lo largo de la sucesión secundaria del bosque, en particular modificó el uso en la altura del refugio a lo largo de la sucesión secundaria. En A. dacnicolor, los resultados concuerdan con lo observado por Ribeiro de Oliveira y Cabral-Eterovick (2010) quienes sugieren que la distribución espacial en anuros sintópicos está más influenciada por las preferencias específicas de las especies que por las oportunidades que pueden tener de migrar hacia nuevos microhábitats. Sin embargo, $S$. fodiens sí migra a nuevos microhábitats por lo que presenta plasticidad en el uso del microhábitat, también sugerida por dichos autores y la cual es una determinante importante en la distribución espacial de los anuros.

Aunque la relación entre el uso de microhábitat por los diferentes estadios de desarrollo en anuros ha recibido poca atención, el sexo además de influir sobre aspectos como la dieta y la competencia, también puede influir en el uso del espacio (Vitt, 2000). En A. dacnicolor se observó que los machos estuvieron más asociados a bosques maduros, mientras que hembras, crías y juveniles estuvieron más asociados al pastizal y el bosque joven. Esta distinción puede resultar de los diferentes requerimientos térmicos en respuesta a las diferencias del tamaño corporal, ya que esta especie presenta dimorfismo sexual, en el cual las hembras son de mayor tamaño que los machos (Duellman, 2001). Mientras más grande es un individuo, el radio superficie/volumen se reduce, conduciendo a tasas menores de desecación (Wygoda, 1988). Así, las hembras de A. dacnicolor que son de mayor tamaño podrían tener tasas menores de evaporación permitiéndoles habitar en sitios más abiertos en comparación con los machos, quienes al tener menor talla y mayor tasa de evaporación ocurren con mayor frecuencia en el bosque maduro donde encuentran mayor cobertura herbácea como arbustos y árboles (Soto-Sandoval, 2013). Aunado a esto, la presencia de charcos y reservorios de agua hechos por el hombre en el paisaje podrían influir en la presencia de hembras, juveniles y crías, ya que favorecen los sitios para ovoposición y reproducción (Suazo-Ortuño, AlvaradoDíaz \& Martínez-Ramos, 2011) así como de regulación de temperatura. Estos resultados pueden fortalecer lo sugerido por Urbina-Cardona et al. (2006, 2012), quienes indican que el uso del espacio puede cambiar en términos de las necesidades térmicas de los individuos conforme estos van avanzando en sus etapas reproductivas, esto se evidencia en la segregación que han tenido los diferentes estadios de desarrollo de $S$. fodiens en este estudio.

Los requerimientos del microhábitat para la mayoría de las especies de anfibios son virtualmente desconocidas lo que limita la implementación de estrategias encaminadas a su conservación en paisajes antropizados (Shoo et al., 2011). Nuestro estudio aporta información relevante sobre el microhábitat utilizado en respuesta a la sucesión secundaria del bosque tropical seco de dos especies sintópicas. Aunque ambas especies han sido reportadas como generalistas por su persistencia en paisajes antropizados (Ramírez-Bautista, 1994; Suazo-Ortuño et al., 2015), los resultados de nuestro estudio sugieren que $A$. dacnicolor es una especie con mayor especialización en el uso del microhábitat que $S$. fodiens, especie que presentó mayor plasticidad ecológica en el uso del microhábitat. Estas diferencias en la respuesta de las especies a la perturbación del hábitat podrían ayudar a explicar porque algunas especies de anfibios son más exitosas en los ambientes antropizados que otras. Por otro lado, también refuerzan la idea que los paisajes con bosques secundarios en distintas 
etapas de sucesión pueden proveer microhábitas adecuados para la permanencia de las especies de anuros.

\section{AGRADECIMIENTOS}

Este estudio forma parte del proyecto la "La biodiversidad y su resiliencia en paisajes modificados por actividades humanas" de la Red Temática Conservación de la Biodiversidad en Ambientes Antropizados PRODEPSEP. Agradecemos a Jorge D. Rangel Orozco y Nancy Lara Uribe por su apoyo logístico en el trabajo de campo, a Héctor Hugo SiliceoCantero por su apoyo en la revisión y comentarios al manuscrito y a la Coordinación de la Investigación Científica de la Universidad Michoacana de San Nicolás de Hidalgo. Los resultados del presente estudio son parte de la tesis de Maestría en Ciencias en Ecología Integrativa de la autora principal, bajo la dirección de Ireri Suazo-Ortuño. La primera autora agradece el apoyo del Programa Nacional de Becas del CONACYT.

\section{RESUMEN}

La perturbación del hábitat puede modificar los microhábitats y por lo tanto tener un marcado efecto en la abundancia y distribución de especies de anuros, en particular en aquellas asociadas a microhábitats específicos. En el presente estudio evaluamos cambios en el uso del microhábitat de dos especies sintópicas de ranas arborícolas, Agalychnis dacnicolor y Smilisca fodiens a lo largo de un gradiente sucesional del bosque tropical seco (BTS). Nuestra hipótesis fue que debido a que estas dos especies son generalistas de hábitat, modificarían el uso del microhábitat en respuesta a la sucesión secundaria del BTS y la temporada climática (TC). Aunque ambas especies se registraron en los bosques secundarios y en los bosques maduros, $A$. dacnicolor prefirió el bosque maduro mientras que $S$. fodiens prefirió los lugares perturbados. Ninguna de las dos especies presentaron diferencias en biomasa entre los estadios sucesionales (ES), ni entre temporadas climáticas. A diferencia de $S$. fodiens la rana $A$. dacnicolor presentó mayores tallas durante la temporada de lluvias. La temperatura corporal de ambas especies varió entre ES y temporada climática. $S$. fodiens presentó una temperatura mas constante entre individuos en los pastizales, mientras que $A$. dacnicolor en los bosques maduros. Las variables ambientales y estructurales del hábitat que influyeron en la presencia de ambas especies dentro de los diferentes ES fueron la temperatura del sustrato, altura del árbol o arbusto donde se encontraba perchando el individuo y los porcentajes de cobertura de hojarasca, herbáceas, arbustos, árboles y suelo desnudo. Las variables que mejor explicaron la biomasa, la longitud hocico-cloaca (LHC) y la temperatura corporal de $S$. fodiens fueron las ramas secas y la temperatura del sustrato y en $A$. dacnicolor la biomasa estuvo mejor explicada por las herbáceas, LHC, la altura del árbol $\mathrm{o}$ arbusto donde la rana perchaba, y la temperatura corporal por la temperatura del sustrato. Ambas especies usaron de manera diferencial las variables ambientales y estructurales del microhábitat conforme los ES aumentaron en edad. Las variables que separaron a las especies fueron la altura del refugio o percha y el porcentaje de árboles en los diferentes ES. A. dacnicolor mantuvo preferencia por los estratos mas bajos de la vegetación, mientras que $S$. fodiens mostró una amplia plasticidad en el uso del microhábitat, en particular modificó el uso en la altura del refugio o percha. Nuestros resultados sugieren que aunque ambas especies han sido consideradas generalistas de hábitat, en los paisajes antropizados $A$. dacnicolor es una especie especialista del microhábitat y $S$. fodiens es una especie con mayor plasticidad ecológica en el uso del microhábitat. Estas diferencias en el uso del microhábitat en respuesta a la perturbación del hábitat pueden ayudar a explicar el éxito de las especies en estos nuevos paisajes, así como la importancia de los bosques secundarios en distintas etapas de sucesión para mantener microhábitats adecuados para la permanencia de las especies en los paisajes antropizados.

Palabras clave: Hylidae, cronosecuencia, características del hábitat, bosques secundarios.

\section{REFERENCIAS}

Blaustein, R. A., Han, A. B., Relyea, R. A., Johnson, T.J. P., Buck, C. J., Gervasi, S. S., \& Kats, B. L. (2011). The complexity of amphibian population declines: understanding the role of cofactor in driving amphibian losses. Annals of the New York Academy of Science, 11223(1), 108-119.

Bradford, C., \& Garcia-Aguayo, A. (1992). Seasonality, predation, and the behaviour of a tropical mainland anole. Journal of Animal Ecology, 61, 717-733.

Burgos, A., \& Mass, J. M. (2004). Vegetation change associated with land-use in tropical dry forest areas of Western Mexico. Agriculture, ecosystems and environment, 104, 475-481.

Bustos-Zagal, M. G., Manjarrez, J., \& Franco, C. R. (2013). Uso de microhábitat y termorregulación en Sceloporus horridus horridus (Wiegman 1939) (Sauria: Phrynosomatidae). Acta Zoológica Mexicana, 29(1), 153-163.

Clarke, K. R., \& Gorley, R. N. (2015). PRIMER v5, v6 \& v7: User manual/tutorial, PRIMER-E, Plymouth UK, 91,192-296. 
Cortés-Gómez, M. A., Castro-Herrera, F., \& Urbina-Cardona, J. N. (2013). Small changes in vegetation structure create great changes in amphibian ensembles in the Colombian Pacific rainforest. Tropical Conservation Science, 6(6), 749-769.

Crump, M. L., \& Scott, Jr. N. J. (1994). Visual Encounter Surveys. In W. R. Heyer, M. A. Donnelly, R. W. McDiarmid, L. A. C. Hayek, \& M. S. Foster (Eds.), Measuring and Monitoring Biological Diversity. Standard Methods for Amphibians (pp. 84-92). Washington DC: Smithsonian Institution Press.

Cuevas, M. F., \& Martori, R. (2007). Diversidad trófica de dos especies sintópicas del género Leptodactylus (Anura: Leptodactylidae) del Sudeste de la provincia de Córdoba, Argentina. Cuadernos Herpetológicos, 21(1), 7-19.

Del Río-García, J., Serrano-Cardozo, H. V., \& RamírezPinilla, M. (2014). Diet and microhabitat use of Bolitoglossa cf. pandi (Caudata: Plethodontidae) from the Cordillera Oriental of Colombia. South American Journal of Herpetology, 9(1), 52-61.

Dirzo, R., Young, S. H., Mooney, A. H., \& Ceballos, G. (Eds.) (2011). Seasonally dry tropical forest: Ecology and conservation. Washington, Covelo, London: Island Press.

Duellman, E. W. (2001). Hylid frogs of Middle America (Expended edition Volume 1). Ithaca, New York: Society for the study of amphibians and reptiles, contributions to herpetology.

Duellman, E. W., \& Trueb, L. (1994). Biology of Amphibians. Baltimore and London: The Johns Hopkins University Press.

Harper, K. A., MacDonald, S. E., Burton, P. J., Chen, J., Brosofske, K. D., Saunders, S. C., Euskirchen, E. S., Roberts, D., Jaiteh, M. S., \& Esseen, P. A. (2005). Edge influence on forest structure and composition in fragmented landscapes. Conservation Biology, 19, 768-782.

Hilje, B., \& Aide, M. T. (2012). Recovery of amphibian species richness and composition in a chronosequence of secondary forest, northeastern Costa Rica. Biological Conservation, 146, 170-176.

Hillers, A., Veith, M., \& Rödel, M. O. (2008). Effects of forest fragmentation and habitat degradation on West African leaf-litter frogs. Conservation Biology, 22(3), 762-772.

Hunter, M. (1996). Habitat degradation and loss. In M. Hunter (Ed.), Fundamentals of Conservation Biology (pp. 179-190). Cambridge, Massachusetts: Blackwell Science.

Isaacs-Cubides, P. J., \& Urbina-Cardona, J. N. (2011). Anthropogenic disturbance and edge effects on anuran assemblages inhabiting cloud forest fragments in Colombia. Naturaleza \& Conservacao, 9(1), 39-46.
Janzen, D. H. (1988). Tropical ecological and biocultural restoration. Science, 239, 243-244.

Joly, P., Miaud, C., Lehmann, A., \& Grolet, O. (2001). Habitat matrix effects on pond occupancy in newts. Conservation Biology, 15, 239-248.

Lott, E. J., \& Atkinson, T. H. (2002). Biodiversidad y fitogeografía de Chamela- Cuixmala, Jalisco. In F. A. Noguera, J. H. Vega, A. N. García, \& M. Quesada (Eds.), Historia Natural de Chamela (pp. 83-97). México, D.F.: Universidad Nacional Autónoma de México.

MacFadden, S. W., \& Capen, D. E. (2002). Avian habitats relationships at multiple scale in a New England forest. Forest Science, 48, 243-253.

Martínez-Coronel, M., \& Pérez-Gutiérrez, M. (2011). Composición de la dieta de Craugastor lineatus (Anura: Craugastoridae) de Chiapas, México. Acta Zoológica Mexicana, 27(2), 215-230.

Menin, M., (2005). Resource use and coexistence of two syntopic hylid frogs (Anura, Hylidae). Revista Brasileira de Zoologia, 22, 61-72.

Meza-Santiago, L. (2014). Estudio de la dieta de Smilisca fodiens en una cronosecuencia de campos abandonados del Bosque Tropical Seco en la Región de Chamela, Jalisco, México (Tesis de Licenciatura). Universidad Michoacana de San Nicolás de Hidalgo, Morelia, Michoacán, México.

Morales, J., \& Sarmiento, L. (2002). Dinámica de los macroinvertebrados edáficos y su relación con la vegetación en una sucesión secundaria en el páramo Venezolano. Ecotrópicos, 15(1), 99-110.

Neckel-Oliveira, S., \& Gascon, C. (2006). Abundance, body size and movement patterns of a tropical treefrog in continuous and fragmented forests in the Brazilian Amazon. Biological Conservation, $128,208-315$.

Pizano, C., \& García, H. (2014). El bosque seco tropical en Colombia. Bogotá, Colombia: Instituto de Investigación de Recursos Biológicos Alexander von Humboldt.

Portillo-Quintero, A. C., \& Sánchez-Azofeita, A. G. (2010). Extent and conservation of tropical dry forest in the Americas. Biological Conservation, 143, 144-155.

Promis, A., Caldentey, J., \& Ibarra, M. (2010). Microclima en el interior de un bosque de Nothofagus pumilio y el efecto de una corta de regeneración. Bosque (Valdivia), 31(2), 129-139.

Ramírez-Bautista, A. (1994). Manual y claves ilustradas de los anfibios y reptiles de la región de Chamela, Jalisco, México. México, D.F.: Cuadernos del Instituto de Biología, Universidad Nacional Autónoma de México. 
Ribeiro de Oliveira, F. F., \& Cabral-Eterovick, P. (2010) Patterns of spatial distribution and microhabitat use by syntopic anuran species along permanent lotic ecosystems in the Cerrado of Southeastern Brazil. Herpetologica, 66(2), 159-171.

Rittenhouse, T. A. G., Harper, E. B., Rehard, L. R., \& Semlitsch, R. D. (2008). The role of microhabitats in the desiccation and survival of anurans in a recently harvested oak-hickory forest. Copeia, 807-814.

Shoo, L. P., Olson, D. H., McMenamin, S. K., Murray, K. A., Van Sluys, M., Donnelly, M. A., ... Hero, J. M. (2011). Engineering a future for amphibians under climate change. Journal of Applied Ecology, 48(2), 487-492.

Soto-Sandoval, Y. (2013). Uso del microhábitat nocturno de dos especies simpátricas (Agalyhcnis dacnicolor y Smilisca fodiens, Anura, Hylidae) en el bosque tropical seco de Chamela, Jalisco, México (Tesis de Licenciatura). Universidad Michoacana de San Nicolás de Hidalgo, Morelia, Michoacán, México.

Suazo-Ortuño, Alvarado-Díaz, J., \& Martínez-Ramos, M. (2011). Riparian areas and conservation of herpetofauna in a tropical dry forest in western Mexico. Biotropica, 43(2), 237-245.

Suazo-Ortuño, I., Alvarado-Díaz, J., Mendoza, E., LópezToledo, L., Lara-Uribe, N., Márquez-Camargo, C., ... Rangel-Orozco, J. D. (2015). High resilience of herpetofaunal communities in a human-modified tropical dry forest landscape in western Mexico. Tropical Conservation Science, 8(2), 396-423.

Todd, B. D., Rothermel, B. B., Reed, R. N., Luhring, T. M., Schlatter, K., Trenkamp, L., \& Gibbons, J. W. (2008). Habitat alteration increases invasive fire ant abundance to the detriment of amphibians and reptiles. Biological Invasion, 10, 539-546.

Toft, C. A. (1985). Resource partitioning in amphibians and reptiles. Copeia, 1985(1), 1-21.

Trejo, I., \& Dirzo, R. (2000). Deforestation in seasonally dry tropical forest: A national and local analysis in Mexico. Biological Conservation, 94, 133-142.

Trilleras, J. M. (2008). Análisis socio-ecológico del manejo, degradación y restauración del bosque tropical seco de la región de Chamela-Cuixmala, México (Tesis de Maestría). Universidad Nacional Autónoma de México, México.

Urbina-Cardona, J. N., Navas, C. A., González, I., GómezMartínez, M. J., Llano-Mejía, J., Medina-Rangel, G. F., \& Blanco, A. (2014). Determinantes de la distribución de los anfibios en el bosque seco tropical de Colombia: herramientas para su conservación. In C. Pizano \& H. García (Eds.), el bosque seco tropical en Colombia ii. Biodiversidad asociada al bosque seco (pp. 163-189). Bogotá Colombia: Instituto Alexander von Humboldt.
Urbina-Cardona, J. N., Reynoso, V. H., \& Dirzo, R. (2012). Spatio-temporal variation in edge effects on the herpetofauna of a Mexican tropical rain forest. In A. D. Bezerra \& T. S. Ferreria (Eds.), Evergreens: Types, Ecology and Conservation (pp. 97-120). New York, USA: Nova Science Publishers, Inc.

Urbina-Cardona, J. N., \& Reynoso, V. H. (2011). Descripción y modelado del microhábitat de los anfibios y reptiles que habitan la selva alta perennifolia de los Tuxtlas. In V. H. Reynoso \& R. I. Coates (Eds.), Avances y Perspectivas en la Investigación de los Bosques Tropicales y sus Alrededores: La Región de Los Tuxtlas (pp. 355-384). México, D. F.: Instituto de Biología, Universidad Nacional Autónoma de México.

Urbina-Cardona, J. N. (2008). Conservation of Neotropical herpetofauna: Research trends and challenges. Tropical Conservation Sciences, 1(4), 359-375.

Urbina-Cardona, J. N., Olivares-Pérez, M., \& Reynoso V. H. (2006). Herpetofauna diversity and microenvironment correlates across the pasture-edge-interior gradient in tropical rainforests fragments in the region of Los Tuxtlas, Veracruz. Biological Conservation, $132,61-75$

Urbina-Cardona, J. N., \& Londoño, M. C. (2003). Distribución de la comunidad de herpetofauna en áreas con diferente grado de perturbación antrópica en la Isla Gorgona, Pacífico Colombiano. Revista de la Academia Colombiana de Ciencias, 27(102), 105-113.

Vitt, L. J. (2000). Ecological consequences of body size in neonatal and small-bodied lizards in the neotropics. Herpetological Monographs, 14, 388-400.

Vitt, L. J., \& Caldwell, J. P. (2014). Herpetology: An Introductory Biology of Amphibians and Reptiles. Syndey, Tokyo: Academic Press.

Vitt, L. J., Ávila-Pires, T. C. S., Esposito, M. C., Sartorius, S. S., \& Zani, P. A. (2007). Ecology of Alopoglossus angulatus and A. atriventris (Squamata, Gymnophthalmidae) in western Amazonia. Phyllomedusa, $6(1), 11-21$

Webb, J. K., \& Shine, R. (1998). Using thermal ecology to predict retreat-site selection by an endangered snake species. Biological Conservation, 86, 233-242.

Wygoda, M. (1988). Adaptive control of water loss resistance in an arboreal frog. Herpetologica, 44, 251-251.

Zúñiga-Vega, J. J., Suárez-Rodríguez, M., Espinoza-Pérez, H., \& Johnson, B. J. (2011). Morphological and reproductive variation among populations of the Pacific molly Poecilia butleri. Journal of Fish Biology, 79(4), 1029-1046. 


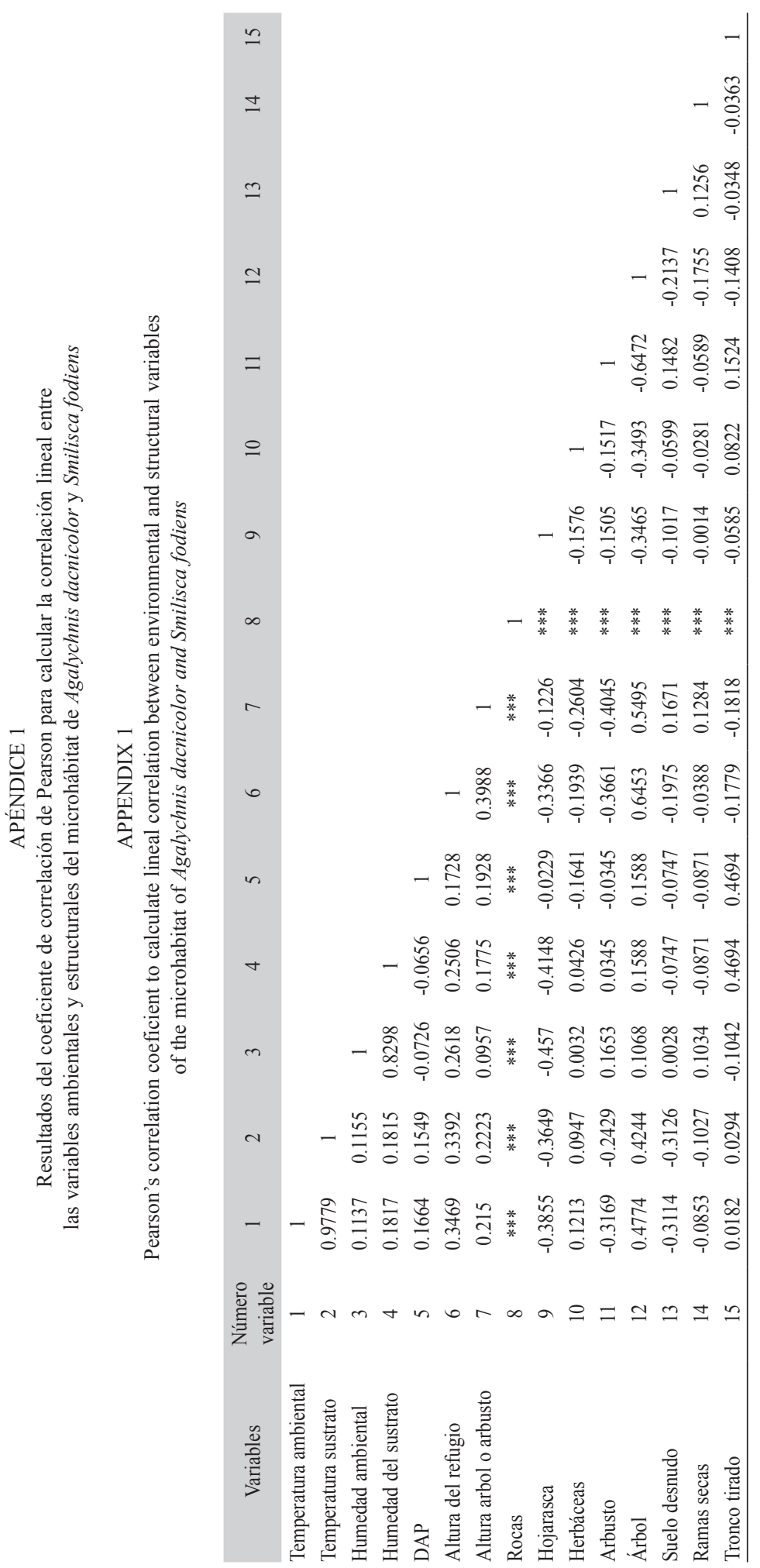




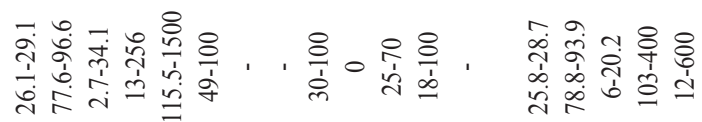

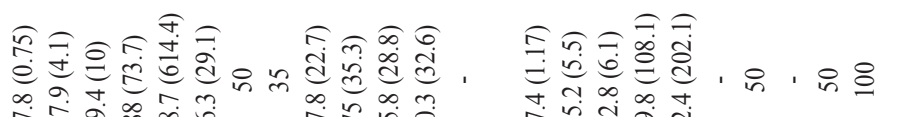

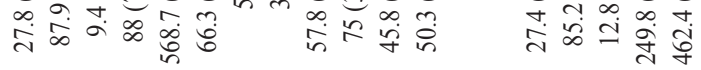

ㄱำ 8 \& 8

ปे

$\stackrel{\circ}{\stackrel{8}{+}}, \frac{8}{7}$

ఏ.

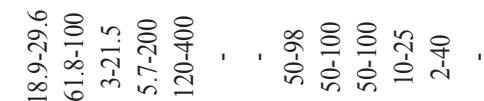

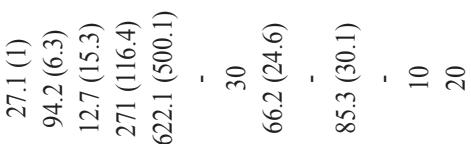

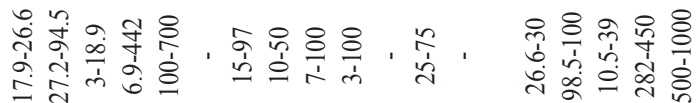

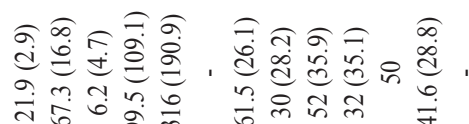

声宅

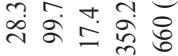

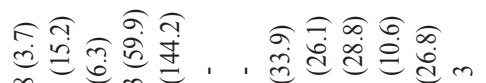

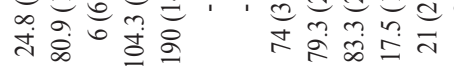

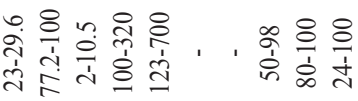

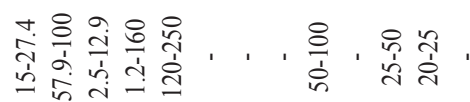

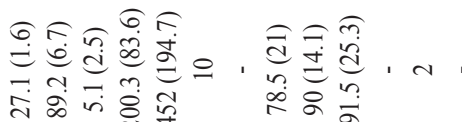

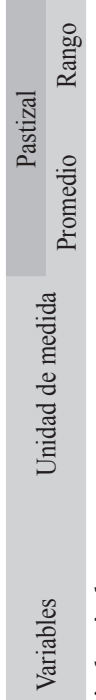

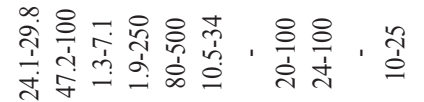

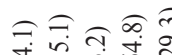

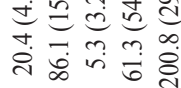

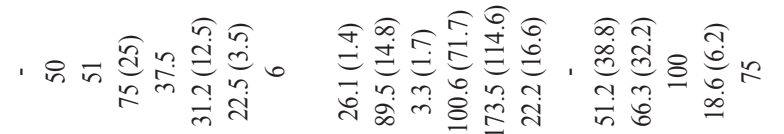

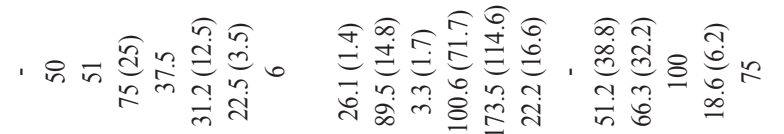

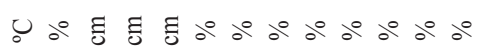

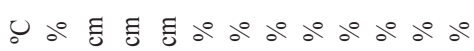

क्ष

:

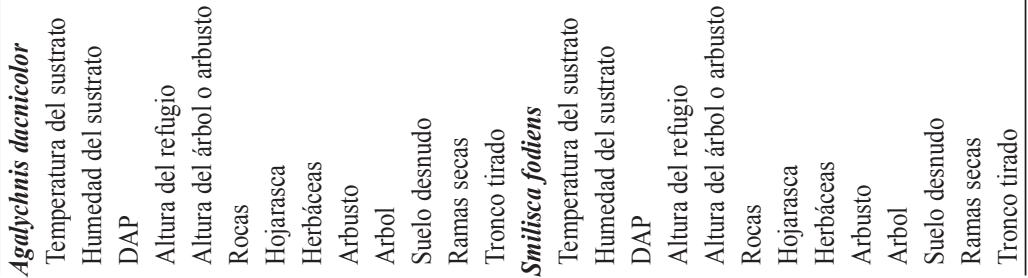




\section{APÉNDICE 3}

Modelos lineales basados en distancias, evaluados a partir del criterio de información de Akaike, clasificados por el número de variable, explicando la biomasa, LHC y la temperatura corporal por la variable utilizada. Agalychnis dacnicolor

\section{APPENDIX 3}

Lineal models based on distances, evaluated by the information criteria of Akaike, classified by the number of variable, explaining biomass, LHC and body temperature by the used variable. Agalychnis dacnicolor

\begin{tabular}{|c|c|c|c|c|c|}
\hline & $\mathrm{AICc}$ & R2 Ajustada & RSS & Número de variables & Variables seleccionadas \\
\hline \multicolumn{6}{|l|}{ Agalychnis dacnicolor } \\
\hline \multicolumn{6}{|l|}{ Biomasa } \\
\hline & 161.09 & 0.20259 & 4853.4 & 1 & 8 \\
\hline & 158.06 & 0.30797 & 4066.8 & 2 & $8 ; 12$ \\
\hline & 157.61 & 0.36067 & 3679.5 & 3 & $7 ; 8 ; 12$ \\
\hline & 154.1 & 0.048342 & 2710.5 & 5 & $1-3 ; 8 ; 11$ \\
\hline & 155.24 & 0.49927 & 2522.2 & 6 & $1-3 ; 7 ; 8 ; 11$ \\
\hline & 155.24 & 0.49927 & 2533.3 & 7 & $1-3 ; 6-8 ; 11$ \\
\hline & 175.51 & 0.4107 & 2349.9 & 12 & Todas \\
\hline \multicolumn{6}{|l|}{ LHC } \\
\hline & 118.76 & 0.42177 & 1238.9 & 1 & 5 \\
\hline & 114.03 & 0.52507 & 982.5 & 2 & $3 ; 5$ \\
\hline & 110.06 & 0.6021 & 793.74 & 3 & $3 ; 5 ; 8$ \\
\hline & 110.03 & 0.69334 & 521.11 & 7 & $1-3 ; 5 ; 10-12$ \\
\hline & 110.03 & 0.69334 & 521.11 & 8 & $1-3 ; 5 ; 6 ; 10-12$ \\
\hline & 125.84 & 0,66276 & 473.41 & 12 & Todas \\
\hline \multicolumn{6}{|l|}{ Temperatura corporal } \\
\hline & -81.774 & 0.67322 & 1.9218 & 1 & 1 \\
\hline & -87.946 & 0.74381 & 1.4547 & 2 & $1 ; 8$ \\
\hline & -95.184 & 0.80688 & 1.0574 & 3 & $1 ; 7 ; 9$ \\
\hline & -96.362 & 0.84468 & 0.7874 & 5 & $1 ; 2 ; 7-9$ \\
\hline & -95.597 & 0.85488 & 0.6024 & 6 & $1 ; 2 ; 4-9 ; 11$ \\
\hline & -95.597 & 0.86499 & 0.6024 & 8 & $1 ; 2 ; 4 ; 5 ; 7-9 ; 11$ \\
\hline & -99.318 & 0.85929 & 0.6849 & 9 & $1 ; 2 ; 4 ; 7 ; 9 ; 11$ \\
\hline & -82.43 & 0.85151 & 0.5721 & 12 & Todas \\
\hline \multicolumn{6}{|l|}{ Smilisca fodiens } \\
\hline \multicolumn{6}{|l|}{ Biomasa } \\
\hline & 243.44 & 0.11004 & 5172.6 & 1 & 14 \\
\hline & 242.15 & 0.15166 & 4832.1 & 2 & $4 ; 14$ \\
\hline & 241.79 & 0.17796 & 4586.6 & 3 & $4 ; 9 ; 14$ \\
\hline & 241.1 & 0.20978 & 4317.3 & 4 & $4 ; 5 ; 9-14$ \\
\hline & 241.1 & 0.20978 & 4317.3 & 5 & $4 ; 5 ; 8 ; 9 ; 14$ \\
\hline & 243.19 & 0.22319 & 4063.5 & 6 & $4 ; 5 ; 9 ; 10 ; 13 ; 14$ \\
\hline & 243.19 & 0.22319 & 4063.5 & 7 & $4 ; 5 ; 8-10 ; 13 ; 14$ \\
\hline & 244.91 & 0.22193 & 3979.6 & 8 & $3-5 ; 7-9 ; 13 ; 14$ \\
\hline & 262.45 & 0.14085 & 4796 & 15 & Todas \\
\hline \multicolumn{6}{|l|}{ LHC } \\
\hline & 166.79 & 0.035447 & 1161.9 & 1 & 14 \\
\hline & 165.49 & 0.06292 & 1106.3 & 2 & $6 ; 14$ \\
\hline & 165.49 & 0.06292 & 1106.3 & 3 & $6 ; 8 ; 14$ \\
\hline & 167.23 & 0.054548 & 1093.4 & 4 & $3 ; 6 ; 8-14$ \\
\hline
\end{tabular}


APÉNDICE 1 (Continuación) / APPENDIX 1 (Continued)

\begin{tabular}{cccccc} 
& AICc & R2 Ajustada & RSS & Número de variables & Variables seleccionadas \\
& 168.6 & 0.054375 & 1070.8 & 5 & $1 ; 2 ; 6 ; 8 ; 14$ \\
Temperatura corporal & 193.12 & 0.092883 & 1000.6 & 15 & Todas \\
& & & & & 2 \\
& -171.93 & 0.12685 & 1.7565 & 2 & $2 ; 10$ \\
& -175.77 & 0.20761 & 1.5622 & 3 & $2 ; 10 ; 14$ \\
& -175.21 & 0.2185 & 1.5093 & 4 & $2 ; 6 ; 10 ; 14$ \\
-174.26 & 0.22461 & 1.4663 & 12 & $2 ; 6 ; 7 ; 10 ; 14$ \\
\hline
\end{tabular}

(1) temperatura del sustrato, (2) humedad ambiental, (3) DAP, (4) altura del refugio o percha, (5) altura del árbol o arbusto, (6) rocas, (7) hojarasca, (8) herbácea, (9) arbusto, (10) suelo desnudo, (11) ramas secas, (12) troncos caídos. Smilisca fodiens: (1) temperatura ambiental, (2) temperatura del sustrato, (3) humedad ambiental, (4) humedad del sustrato, (5) DAP, (6) altura del refugio o percha, (7) altura del árbol o arbusto, (8) rocas, (9) hojarasca, (10) herbácea, (11) arbusto, (12) árbol, (13) suelo desnudo, (14) ramas secas, (15) troncos caídos.

(1) substrate temperature, (2) air humidity, (3) DAP, (4) height of refuge or perch, (5) height of tree or shrub, (6) rocks, (7) litter, (8) herbs, (9) shrubs, (10) bare ground, (11) dry branches, (12) fallen logs. Smilisca fodiens: (1) air temperatura, (2) substrate temperatura, (3) air humidity, (4) substrate humidity, (5) DAP, (6) height of refuge or perch, (7) height of tree or brush, (8)rocks, (9) litter, (10) herbs, (11) brush, (12) tree, (13) bare soil, (14) dry branches, (15) fallen logs. 